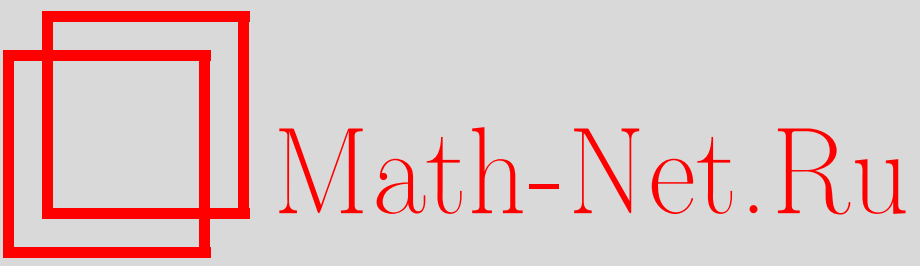

А. В. Тарасов, Стабилизаторы некоторых семейств булевых функций от $n$ переменных, образующих Галуа-замкнутые подалгебры алгебры Шефера. II, $M a$ тем. вопр. криптогр., 2017, том 8, выпуск 4, 135-156

DOI: https://doi.org/10.4213/mvk237

Использование Общероссийского математического портала Math-Net.Ru подразумевает, что вы прочитали и согласны с пользовательским соглашением

http://www . mathnet.ru/rus/agreement

Параметры загрузки:

IP : 54.92 .164 .108

26 апреля 2023 г., 03:22:41 
МАТЕМАТИЧЕСКИЕ ВОПРОСЫ КРИПТОГРАФИИ

2017 T. 8 № 4 C. 135-156

УДК 519.716 .5

\title{
Стабилизаторы некоторых семейств булевых функций от $n$ переменных, образующих Галуа-замкнутые подалгебры алгебры Шефера. II
}

\author{
A. B. Tapacoв \\ Московский государственный университет информационных технологий, \\ радиотехники и электроники, Москва
}

Получено 11.V.2017

\begin{abstract}
Аннотация. В статье завершено описание групп подстановок множества двоичных векторов длины $n$, стабилизирующих классы булевых функций от $n$ переменных из Галуа-замкнутых подалгебр алгебры Шефера.

Ключевые слова: булева функция, классы Шефера, классы Поста, соответствия Галуа, группа подстановок

The stabilizers of certain families of Boolean functions of $n$ variables that form a Galois-closed subalgebra of the Schaefer algebra. II

A. V. Tarasov

Moscow State University of Information Technologies, Radio Engineering and Electronics, Moscow
\end{abstract}

\begin{abstract}
We complete the description of permutation groups over the set of binary $n$-dimensional vectors which stabilizes classes of Boolean functions of $n$ variables from the Galois-closed subalgebras of the Schaefer algebra.
\end{abstract}

Key words: Boolean function, Schaefer classes, Post classes, Galois connections, permutation group 


\section{Введение}

В статье завершено начатое в [1-3] описание максимальных групп подстановок множества двоичных $n$-мерных векторов, стабилизирующих Галуазамкнутые классы булевых функций, которые образуют подалгебры алгебры Шефера.

Важной характеристикой класса булевых функций от $n$ переменных является группа преобразований множества $n$-мерных двоичных векторов, стабилизирующих данный класс (стабилизатор множества функций). Вместе с тем представляют интерес для изучения классы булевых функций, порождающие эффективно решаемые системы булевых уравнений. К таким классам относятся классы 0-выполнимых, 1-выполнимых, мультиаффинных, биюнктивных слабо положительных и слабо отрицательных функций, описанные, например, в монографии [11]. В [1] изучены стабилизаторы классов биюнктивных, мультиаффинных, слабо положительных и слабо отрицательных булевых функций от $n$ переменных.

Указанные классы булевых функций и их пересечения обладают рядом свойств, позволяющих установить соответствия Галуа между ними и замкнутыми классами Поста. В [3] на основе результатов работ [4-7] введена универсальная алгебра Шефера, основное множество которой составляет множество всех булевых функций, а множество операций состоит из операций коньюнкции функций, перестановок переменных и проектирования функции по переменной. Решетка подалгебр этой алгебры состоит в основном из классов Шефера и их всевозможных пересечений. В [3] показана связь этой алгебры с итеративной алгеброй Поста, осуществляемая посредством пары соответствий Галуа. Выделены подалгебры алгебры Шефера, являющиеся Галуа-замкнутыми классами.

В [2] приведено описание стабилизаторов ряда множеств булевых функций от $n$ переменных, лежащих в некоторых Галуа-замкнутых подалгебрах алгебры Шефера.

\section{1. Основные определения, обозначения и факты}

Обозначения введем в соответствии с работами $[1-4,11]$. Пусть:

$V_{n}$ - множество $n$-мерных двоичных векторов,

$1_{n}=(\underbrace{1, \ldots, 1}_{n}), 0_{n}=(\underbrace{0, \ldots, 0}_{n})$,

$\|\alpha\|$ - вес Хемминга вектора $\alpha \in V_{n}$,

$P_{2}-$ множество всех булевых функций,

$P_{2}(n)$ - множество булевых функций от $n$ переменных, 
$E_{f}=\left\{\alpha \in V_{n}: f(\alpha)=1\right\}$ - носитель (множество выполняющих векторов) функции $f \in P_{2}(n)$,

$\left\|f\left(x_{1}, \ldots, x_{n}\right)\right\|-$ вес булевой функции $f \in P_{2}(n)$.

Для произвольного класса функций $K \subset P_{2}$ обозначим

$$
K(n)=K \cap P_{2}(n)=\left\{f\left(x_{1}, \ldots, x_{n}\right) \in K\right\} .
$$

Введем важные классы функций. Булева функция $f\left(x_{1}, \ldots, x_{n}\right)$ называется:

1) 0-выполнимой, если $f(0, \ldots, 0)=1$,

2) 1-выполнимой, если $f(1, \ldots, 1)=1$,

3) мультиаффинной, если $f \equiv 1$ или существует представление $f$ в виде

$$
f\left(x_{1}, \ldots, x_{n}\right) \equiv \bigwedge_{i=1}^{t} l_{i}\left(x_{1}, \ldots, x_{n}\right)
$$

где $l_{1}\left(x_{1}, \ldots, x_{n}\right), \ldots, l_{t}\left(x_{1}, \ldots, x_{n}\right)$ - аффинные функции,

4) биюнктивной, если $f \equiv 1$ или существует представление функции $f$ в виде 2-КНФ

$$
f\left(x_{1}, \ldots, x_{n}\right)=\bigwedge_{j \in J} x_{j}^{b_{j}} \bigwedge_{i=1}^{t}\left(x_{s_{i 1}}^{a_{i 1}} \vee x_{s_{i 2}}^{a_{i 2}}\right)
$$

5) слабо положительной, если $f \equiv 1$ или существует представление $f$ в виде КНФ

$$
f\left(x_{1}, \ldots, x_{n}\right) \equiv \bigwedge_{i=1}^{t}\left(x_{s_{i 1}}^{a_{i 1}} \vee x_{s_{i 2}} \vee \cdots \vee x_{s_{i k_{i}}}\right), \quad k_{i} \geqslant 1, \quad i=\overline{1, t}
$$

6) слабо отрищательной, если $f \equiv 1$ или существует представление $f$ в виде КНФ

$$
f\left(x_{1}, \ldots, x_{n}\right) \equiv \bigwedge_{i=1}^{t}\left(x_{s_{i 1}}^{a_{i 1}} \vee \bar{x}_{s_{i 2}} \vee \cdots \vee \bar{x}_{s_{i k_{i}}}\right), \quad k_{i} \geqslant 1, \quad i=\overline{1, t} .
$$

Множества всех функций классов 1)-6) обозначим соответственно $0-S$, $1-S, A, B i, W P, W N$. Данные классы называются классами Шефера. Формулы (1)-(4) для функций из классов $A, B i, W P, W N$ будем называть приведенными представлениями.

В [11] показано, что именно эти классы порождают системы уравнений с полиномиально разрешимой задачей распознавания совместности. Более того, классы $A, B i, W P, W N$ являются полиномиально решаемыли, т. е. в случае совместности порожденной системы существует полиномиальная процедура построения решений системы. 
Следуя [2], булеву функцию $f\left(x_{1}, \ldots, x_{n}\right)$ назовем $C$-замкнутой, если выполнено равенство

$$
f\left(x_{1}, \ldots, x_{n}\right) \equiv f\left(\bar{x}_{1}, \ldots, \bar{x}_{n}\right) .
$$

Класс всех таких функций обозначим $C L$.

Через $W P_{m, 2}, \quad m \geqslant 2$, обозначим подкласс класса слабо положительных функций, элементы которого представимы в виде

$$
f\left(x_{1}, \ldots, x_{n}\right)=\bigwedge_{i=1}^{t}\left(\bar{x}_{s_{i, 1}} \vee x_{s_{i, 2}}\right) \bigwedge_{j=1}^{p}\left(x_{r_{j, 1}} \vee \cdots \vee x_{r_{j, m}}\right) .
$$

Аналогичным образом введем класс $W N_{m, 2}$. В качестве их естественных расширений введем классы

$$
W P_{\infty, 2}=\bigcup_{m=0}^{\infty} W P_{m, 2}, \quad W N_{\infty, 2}=\bigcup_{m=0}^{\infty} W N_{m, 2} .
$$

Класс булевых монотонных функций обозначим через Mon, а класс антимонотонных функций - через $\overline{M o n}$.

Для векторов $\alpha, \beta \in V_{n}$ через $\alpha \vee \beta, \alpha \wedge \beta$ (или $\alpha \cdot \beta$ ), $\alpha \oplus \beta$ обозначим векторы, получающиеся из векторов $\alpha$ и $\beta$ покоординатным применением соответствующих операций. Определим далее через $v$ тернарное преобразование пространства $V_{n}$, задаваемое равенством

$$
v(\alpha, \beta, \gamma)=(\alpha \wedge \beta) \vee(\alpha \wedge \gamma) \vee(\beta \wedge \gamma), \quad \alpha, \beta, \gamma \in V_{n} .
$$

Для произвольного класса функций $K \subset P_{2}$ положим

$$
K^{0}=K \cap 0-S, K^{1}=K \cap 1-S, K^{01}=K \cap 0-S \cap 1-S, K(n)=K \cap P_{2}(n) .
$$

Известны следующие критерии мультиаффинности, биюнктивности, слабой положительности и слабой отрицательности.

Теорема 1 ([11]). Для функиии $f\left(x_{1}, \ldots, x_{n}\right) \in P_{2}(n)$ верны следующие утверждения:

1) $f\left(x_{1}, \ldots, x_{n}\right) \in A(n)$ тогда и только тогда, когда для любых трех векторов $\alpha, \beta, \gamma \in E_{f}$ вектор $\delta=\alpha \oplus \beta \oplus \gamma \in E_{f}$,

2) $f\left(x_{1}, \ldots, x_{n}\right) \in B i(n)$ тогда и только тогда, когда для любых трех векторов $\alpha, \beta, \gamma \in E_{f}$ вектор $\delta=v(\alpha, \beta, \gamma) \in E_{f}$,

3) $f\left(x_{1}, \ldots, x_{n}\right) \in W P(n)$ тогда и только тогда, когда для любых двух векторов $\alpha, \beta \in E_{f}$ вектор $\delta=\alpha \vee \beta \in E_{f}$,

4) $f\left(x_{1}, \ldots, x_{n}\right) \in W N(n)$ тогда и только тогда, когда для любых двух векторов $\alpha, \beta \in E_{f}$ вектор $\delta=\alpha \wedge \beta \in E_{f}$. 
Данная теорема лежит в основе построения соответствий Галуа для системы замкнутых классов Поста и системы всевозможных пересечений классов $0-S, 1-S, A, B i, W P, W N, C L$, а также их некоторых подклассов (см. [3-7]).

Описание этих классов функций приведено в следующей таблице.

Таблица 1

\begin{tabular}{|c|c|c|}
\hline № & Класс & Функции класса \\
\hline 1 & $P_{2}$ & Класс всех булевых функций \\
\hline 2 & $0-S$ & $f(0, \ldots, 0)=1$ \\
\hline 3 & $1-S$ & $f(1, \ldots, 1)=1$ \\
\hline 4 & $C L$ & Класс $C$-замкнутых функций \\
\hline 5 & $0-S \cap 1-S$ & $f(0, \ldots, 0)=f(1, \ldots, 1)=1$ \\
\hline 6 & $C L^{1}$ & $C L \cap 0-S \cap 1-S, f\left(x_{1}, \ldots, x_{n}\right)=f\left(\bar{x}_{1}, \ldots, \bar{x}_{n}\right), f(1, \ldots, 1)=1$ \\
\hline 7 & $A$ & Класс мультиаффинных функций вида (1) \\
\hline 8 & $A^{0}$ & $f\left(x_{1}, \ldots, x_{n}\right)=\bigwedge_{i=1}^{t}\left(x_{s_{j, 1}} \oplus \cdots \oplus x_{s_{i, k_{i}}} \oplus 1\right)$ \\
\hline 9 & $A^{1}$ & $\begin{array}{l}f\left(x_{1}, \ldots, x_{n}\right)=\bigwedge_{i=1}^{t}\left(x_{s_{j, 1}} \oplus \cdots \oplus x_{s_{i, k_{i}}}\right) \bigwedge_{j=1}^{p}\left(x_{r_{j, 1}} \oplus \cdots \oplus x_{r_{j, q_{j}}}\right) \text {, где } k_{i} \\
\text { нечетны, а } q_{j} \text { четны, } i=\overline{1, t}, j=\overline{1, p}\end{array}$ \\
\hline 10 & $B i$ & Класс биюнктивных функций (2) \\
\hline 11 & $M_{3}$ & $W P \cap W N, f\left(x_{1}, \ldots, x_{n}\right)=\bigwedge_{i \in I} x_{i} \bigwedge_{j \in J} \bar{x}_{j} \bigwedge_{l=1}^{t}\left(\bar{x}_{s_{l, 1}} \vee x_{s_{l, 2}}\right)$ \\
\hline 12 & $M_{3}^{0}$ & $M_{3}^{0}=M_{4}^{0}, f\left(x_{1}, \ldots, x_{n}\right)=\bigwedge_{j \in J} \bar{x}_{j} \bigwedge_{l=1}^{t}\left(\bar{x}_{s_{l, 1}} \vee x_{s_{l, 2}}\right)$ \\
\hline 13 & $M_{3}^{1}$ & $M_{3}^{1}=M_{4}^{1}, f\left(x_{1}, \ldots, x_{n}\right)=\bigwedge_{i \in I} x_{i} \bigwedge_{l=1}^{t}\left(\bar{x}_{s_{l, 1}} \vee x_{s_{l, 2}}\right)$ \\
\hline 14 & $M_{3}^{01}$ & $M_{3}^{01}=M_{4}^{01}=M_{5}^{01}=B i^{01}, f\left(x_{1}, \ldots, x_{n}\right)=\bigwedge_{l=1}^{t}\left(\bar{x}_{s_{l, 1}} \vee x_{s_{l, 2}}\right)$ \\
\hline 15 & $M_{1}$ & $A \cap W P, f\left(x_{1}, \ldots, x_{n}\right)=\bigwedge_{i \in I} x_{i} \bigwedge_{j \in J} \bar{x}_{j} \bigwedge_{l=1}^{t}\left(x_{s_{l, 1}} \oplus x_{s_{l, 2}} \oplus 1\right)$ \\
\hline 16 & $M_{1}^{0}$ & $M_{1}^{0}=M_{2}^{0}, f\left(x_{1}, \ldots, x_{n}\right)=\bigwedge_{j \in J} \bar{x}_{j} \bigwedge_{l=1}^{t}\left(x_{s_{l, 1}} \oplus x_{s_{l, 2}} \oplus 1\right)$ \\
\hline 17 & $M_{1}^{1}$ & $M_{1}^{1}=M_{2}^{1}, f\left(x_{1}, \ldots, x_{n}\right)=\bigwedge_{i \in I} x_{i} \bigwedge_{l=1}^{t}\left(x_{s_{l, 1}} \oplus x_{s_{l, 2}} \oplus 1\right)$ \\
\hline 18 & $W P$ & Класс слабо положительных функций (3) \\
\hline 19 & $W P^{0}$ & $f\left(x_{1}, \ldots, x_{n}\right)=\bigwedge_{j \in J} \bar{x}_{j} \bigwedge_{l=1}^{t}\left(\bar{x}_{s_{l 1}} \vee x_{s_{l 2}} \vee \cdots \vee x_{s_{l k_{l}}}\right)$ \\
\hline 20 & $W P^{1}$ & $f\left(x_{1}, \ldots, x_{n}\right)=\bigwedge_{i \in I} x_{i} \bigwedge_{l=1}^{t}\left(x_{s_{l 1}}^{a_{l}} \vee x_{s_{l 2}} \vee \cdots \vee x_{s_{l k_{l}}}\right), 2 \leqslant k_{l} \leqslant n, l=\overline{1, t}$ \\
\hline 21 & $W P^{01}$ & $f\left(x_{1}, \ldots, x_{n}\right)=\bigwedge_{l=1}^{t}\left(\bar{x}_{s_{l 1}} \vee x_{s_{l 2}} \vee \cdots \vee x_{s_{l k_{l}}}\right), 2 \leqslant k_{l} \leqslant n, l=\overline{1, t}$ \\
\hline 22 & $W P_{\infty, 2}$ & $f\left(x_{1}, \ldots, x_{n}\right)=\bigwedge_{i=1}^{t}\left(\bar{x}_{r_{l 1}} \vee x_{r_{i 2}}\right) \bigwedge_{l=1}^{t}\left(x_{s_{l 1}} \vee \cdots \vee x_{s_{l k_{l}}}\right)$ \\
\hline 23 & $\begin{array}{l}W P_{m, 2} \\
\quad m \geqslant 2\end{array}$ & $f\left(x_{1}, \ldots, x_{n}\right)=\bigwedge_{i \in I} x_{i}^{a_{i}} \bigwedge_{j=1}^{t}\left(\bar{x}_{r_{j 1}} \vee x_{r_{j 2}}\right) \bigwedge_{l=1}^{p}\left(x_{s_{l 1}} \vee \cdots \vee x_{s_{l m}}\right)$ \\
\hline
\end{tabular}


Таблица 1 (продолжение)

\begin{tabular}{|c|c|c|}
\hline 24 & $W N$ & Класс слабо отрицательных функций вида (4) \\
\hline 25 & $W N^{0}$ & $f\left(x_{1}, \ldots, x_{n}\right)=\bigwedge_{i \in I} \bar{x}_{i} \bigwedge_{l=1}^{t}\left(x_{s_{l 1}}^{a_{l}} \vee \bar{x}_{s_{l 2}} \vee \cdots \vee \bar{x}_{s_{l k_{l}}}\right), 2 \leqslant k_{l} \leqslant n, l=\overline{1, t}$ \\
\hline 26 & $W N^{1}$ & $f\left(x_{1}, \ldots, x_{n}\right)=\bigwedge_{i \in I} x_{i} \bigwedge_{l=1}^{t}\left(x_{s_{l 1}} \vee \bar{x}_{s_{l 2}} \vee \cdots \vee \bar{x}_{s_{l k_{l}}}\right), 2 \leqslant k_{l} \leqslant n, l=\overline{1, t}$ \\
\hline 27 & $W N^{01}$ & $f\left(x_{1}, \ldots, x_{n}\right)=\bigwedge_{l=1}^{t}\left(x_{s_{l 1}} \vee \bar{x}_{s_{l 2}} \vee \cdots \vee \bar{x}_{s_{l k_{l}}}\right), 2 \leqslant k_{l} \leqslant n, l=\overline{1, t}$ \\
\hline 28 & $W N_{\infty, 2}$ & $f\left(x_{1}, \ldots, x_{n}\right)=\bigwedge_{i=1}^{t}\left(\bar{x}_{r_{l 1}} \vee x_{r_{i 2}}\right) \bigwedge_{l=1}^{t}\left(\bar{x}_{s_{l 1}} \vee \cdots \vee \bar{x}_{s_{l k_{l}}}\right)$ \\
\hline 29 & $\begin{array}{l}W N_{m, 2} \\
\quad m \geqslant 2\end{array}$ & $f\left(x_{1}, \ldots, x_{n}\right)=\bigwedge_{i \in I} x_{i}^{a_{i}} \bigwedge_{j=1}^{t}\left(\bar{x}_{r_{j 1}} \vee x_{r_{j 2}}\right) \bigwedge_{l=1}^{p}\left(\bar{x}_{s_{l 1}} \vee \cdots \vee \bar{x}_{s_{l m}}\right)$ \\
\hline 30 & $A^{01}$ & $\begin{array}{l}A \cap 0-S \cap 1-S, f\left(x_{1}, \ldots, x_{n}\right)= \\
=\bigwedge_{i=1}^{t}\left(x_{s_{i, 1}} \oplus x_{s_{i, 2}} \oplus \cdots \oplus x_{s_{k_{i}}} \oplus 1\right), \text { где } k_{i} \text { четны }\end{array}$ \\
\hline 31 & $M_{8}$ & $\begin{array}{l}A \cap C L, f\left(x_{1}, \ldots, x_{n}\right)= \\
=\bigwedge_{i=1}^{t}\left(x_{s_{i, 1}} \oplus x_{s_{i, 2}} \oplus \cdots \oplus x_{s_{i_{i}}} \oplus a_{i}\right), \text { где } k_{i} \text { четны }\end{array}$ \\
\hline 32 & $M_{2}$ & $B i \cap A, f\left(x_{1}, \ldots, x_{n}\right)=\bigwedge_{i \in I} x_{i}^{a_{i}} \bigwedge_{j=1}^{m}\left(x_{s_{j, 1}} \oplus x_{s_{j, 2}} \oplus b_{j}\right)$ \\
\hline 33 & $M_{7}$ & $B i \cap C L, f\left(x_{1}, \ldots, x_{n}\right)=\bigwedge_{i=1}^{t}\left(x_{s_{i, 1}} \oplus x_{s_{i, 2}} \oplus a_{i}\right)$ \\
\hline 34 & $M_{1}^{01}$ & $W N \cap C L=W P \cap C L, f\left(x_{1}, \ldots, x_{n}\right)=\bigwedge_{i=1}^{t}\left(x_{s_{i, 1}} \oplus x_{s_{i, 2}} \oplus 1\right)$ \\
\hline 35 & $M_{9}$ & $\begin{array}{l}f\left(x_{1}, \ldots, x_{n}\right)=\bigwedge_{i \in I} \bar{x}_{i} \bigwedge_{j \in J}\left(x_{s_{j, 1}} \oplus x_{s_{j, 2}} \oplus 1\right) \wedge g\left(x_{1}, \ldots, x_{n}\right), \text { где } g \in \text { Mon } \\
\text { и несущественно зависит от переменных } x_{i}, i \in I\end{array}$ \\
\hline 36 & $M_{9}^{1}$ & $\begin{array}{l}M_{9} \cap 1-S, f\left(x_{1}, \ldots, x_{n}\right)= \\
=\bigwedge_{j \in J}\left(x_{s_{j, 1}} \oplus x_{s_{j, 2}} \oplus 1\right) \wedge g\left(x_{1}, \ldots, x_{n}\right), \text { где } g \in \text { Mon }\end{array}$ \\
\hline 37 & Mon' $^{\prime}$ & $f\left(x_{1}, \ldots, x_{n}\right)=f_{1}\left(x_{1}, \ldots, x_{n}\right) \wedge f_{2}\left(x_{1}, \ldots, x_{n}\right)$, где $f_{1} \in M_{1}^{01}, f_{2} \in$ Mon \\
\hline 38 & $\begin{array}{r}\operatorname{Mon}_{m}^{\prime} \\
\quad m \geqslant 2\end{array}$ & $f\left(x_{1}, \ldots, x_{n}\right)=\bigwedge_{i=1}^{t}\left(x_{s_{i, 1}} \oplus x_{s_{i, 2}} \oplus 1\right) \bigwedge_{j=1}^{p}\left(x_{r_{j, 1}} \vee \cdots \vee x_{r_{j, m}}\right)$ \\
\hline 39 & $\begin{array}{l}M_{9, m} \\
\quad m \geqslant 2\end{array}$ & $f\left(x_{1}, \ldots, x_{n}\right)=\bigwedge_{l \in I} \bar{x}_{l} \bigwedge_{i=1}^{t}\left(x_{s_{i, 1}} \oplus x_{s_{i, 2}} \oplus 1\right) \bigwedge_{j=1}^{p}\left(x_{r_{j, 1}} \vee \cdots \vee x_{r_{j, m}}\right)$ \\
\hline 40 & $\begin{array}{l}W P_{m, 2}^{1} \\
\quad m \geqslant 2\end{array}$ & $f\left(x_{1}, \ldots, x_{n}\right)=\bigwedge_{i=1}^{t}\left(\bar{x}_{s_{i, 1}} \vee x_{s_{i, 2}}\right) \bigwedge_{j=1}^{p}\left(x_{r_{j, 1}} \vee \cdots \vee x_{r_{j, m}}\right)$ \\
\hline 41 & $M_{10}$ & $\begin{array}{l}f\left(x_{1}, \ldots, x_{n}\right)=\bigwedge_{j \in J} x_{j} \bigwedge_{i=1}^{t}\left(x_{s_{i, 1}} \oplus x_{s_{i, 2}} \oplus 1\right) \wedge g\left(x_{1}, \ldots, x_{n}\right), \text { где } g \in \\
\overline{M o n} \text { и несущественно зависит от переменных } x_{j}, j \in J\end{array}$ \\
\hline 42 & $M_{10}^{0}$ & $\begin{array}{l}f\left(x_{1}, \ldots, x_{n}\right)=\bigwedge_{i=1}^{t}\left(x_{s_{i, 1}} \oplus x_{s_{i, 2}} \oplus 1\right) \wedge g\left(x_{1}, \ldots, x_{n}\right), \text { где } g \in \overline{M o n} \text { и } \\
\text { несущественно зависит от переменных } x_{j}, j \in J\end{array}$ \\
\hline
\end{tabular}


Таблица 1 (продолжение)

\begin{tabular}{|c|c|c|c|}
\hline 43 & $\overline{\text { Mon' }^{\prime}}$ & $f\left(x_{1}, \ldots, x_{n}\right)=f_{1}\left(x_{1}, \ldots, x_{n}\right) \wedge f_{2}\left(x_{1}, \ldots, x_{n}\right)$, где $f_{1} \in M_{1}^{01}, f_{2} \in \overline{M o n}$ \\
\hline 44 & $\begin{array}{c}{\overline{M o n^{\prime}}}_{m}, \\
m \geqslant 2\end{array}$ & $f\left(x_{1}, \ldots, x_{n}\right)=\bigwedge_{i=1}^{t}\left(x_{s_{i, 1}} \oplus x_{s_{i, 2}} \oplus 1\right) \bigwedge_{j=1}^{p}\left(\bar{x}_{r_{j, 1}} \vee \cdots \vee \bar{x}_{r_{j, m}}\right)$ \\
\hline 45 & $\begin{array}{c}M_{10, m}, \\
m \geqslant 2\end{array}$ & $f\left(x_{1}, \ldots, x_{n}\right)=\bigwedge_{l \in I} x_{l} \bigwedge_{i=1}^{t}\left(x_{s_{i, 1}} \oplus x_{s_{i, 2}} \oplus 1\right) \bigwedge_{j=1}^{p}\left(\bar{x}_{r_{j, 1}} \vee \cdots \vee \bar{x}_{r_{j, m}}\right)$ \\
\hline 46 & $\begin{array}{c}W N_{m, 2}^{0}, \\
m \geqslant 2\end{array}$ & $f\left(x_{1}, \ldots, x_{n}\right)=\bigwedge_{i=1}^{t}\left(\bar{x}_{s_{i, 1}} \vee x_{s_{i, 2}}\right) \bigwedge_{j=1}^{p}\left(\bar{x}_{r_{j, 1}} \vee \cdots \vee \bar{x}_{r_{j, m}}\right)$ \\
\hline
\end{tabular}

Заметим, что во введенных обозначениях $M_{4}=B i \cap W P=W P_{2,2}$ и $M_{5}=B i \cap W N=W N_{2,2}$.

Введем ряд теоретико-групповых обозначений:

$\operatorname{Sym}(X)$ - симметрическая группа подстановок на конечном множестве $X$,

$\varepsilon$ - тождественная подстановка на соответствующем множестве,

$S_{n}=\operatorname{Sym}(\{1,2, \ldots, n\})$,

$\operatorname{Sym}\left(V_{n}\right)$ - симметрическая группа подстановок на множестве $V_{n}$ (изоморфна симметрической группе $\left.S_{2^{n}}\right)$,

$<-$ отношение «быть подгруппой» или «быть подпространством», в зависимости от контекста,

$\operatorname{dim} L-$ размерность подпространства или многообразия $L$,

$\langle M\rangle-$ подгруппа, порожденная множеством элементов $M$ (в произвольной группе),

$\widetilde{S}_{n}<\operatorname{Sym}\left(V_{n}\right)-$ группа, индуцированная действием группы перестановок координат векторов из $V_{n}$ (изоморфна симметрической группе $S_{n}$ ); она состоит из всех подстановок $\widetilde{s}$, задаваемых равенством

$$
\widetilde{s}\left(\left(a_{1}, \ldots, a_{n}\right)\right)=\left(a_{s(1)}, \ldots, a_{s(n)}\right), \quad s \in S_{n},
$$

$\Sigma_{n}$ - группа сдвигов, изоморфная пространству $V_{n}$, состоит из всех подстановок $\widetilde{\beta}, \beta=\left(b_{1}, . ., b_{n}\right) \in V_{n}$, где

$$
\widetilde{\beta}\left(\left(a_{1}, \ldots, a_{n}\right)\right)=\left(a_{1} \oplus b_{1}, \ldots, a_{n} \oplus b_{n}\right),
$$

$Q_{n}=\left\langle\widetilde{S}_{n}, \Sigma_{n}\right\rangle-$ группа Джевонса, равна полупрямому произведению $\widetilde{S}_{n} \lambda \Sigma_{n}$

$C S_{n}=\left\langle\widetilde{S}_{n}, \widetilde{1}_{n}\right\rangle-$ подгруппа группы Джевонса, порождаемая группой $\widetilde{S}_{n}$ и подстановкой $\widetilde{1}_{n}$, инвертирующей векторы из $V_{n}$,

$\overline{C S_{n}}=\left\langle C S_{n},\left(0_{n}, 1_{n}\right)\right\rangle-$ группа, порожденная группой $C S_{n}$ и транспозицией $\left(0_{n}, 1_{n}\right)$, 
$G L_{n}-$ полная линейная группа на пространстве $V_{n}$,

$A G L_{n}$ - полная аффинная группа на пространстве $V_{n}$,

$H_{n}$ - группа инволюций, порожденная транспозициями вида $(\alpha, \bar{\alpha}), \alpha \in V_{n}$,

$\bar{S}_{2^{n}-2}=\left\langle\operatorname{Sym}\left(V_{n} \backslash\left\{0_{n}, 1_{n}\right\}\right),\left(0_{n}, 1_{n}\right)\right\rangle$,

$T_{n}$ - двоичная $n \times n$-матрица, в которой элементы главной диагонали и последней строки равны единице, а остальные - нулю:

$$
T_{n}=\left(\begin{array}{cccc}
1 & 0 & \ldots & 0 \\
0 & 1 & \ldots & 0 \\
\vdots & & \ddots & \vdots \\
1 & 1 & \ldots & 1
\end{array}\right)
$$

$R_{n}^{(1)}$ - подгруппа группы $G L_{n}$, порожденная подстановочными матрицами и матрицей $T_{n}$ (см. [10]),

$$
A R_{n}^{(1)}=\left\langle R_{n}^{(1)}, \Sigma_{n}\right\rangle=R_{n}^{(1)} \lambda \Sigma_{n}-\text { соответствующая подгруппа группы }
$$
$A G L_{n}$

изоморфизм групп обозначим символом $\cong$.

Введенные группы естественным образом задают свое действие на множестве функций $P_{2}(n):$ если $s \in \operatorname{Sym}\left(V_{n}\right)$, то для функции $f\left(x_{1}, \ldots, x_{n}\right) \in$ $P_{2}(n)$ определим функцию $s(f)=f^{s}\left(x_{1}, \ldots, x_{n}\right)$, задаваемую равенством $E_{f s}=s\left(E_{f}\right)$, или, что равносильно, равенством $f^{s}\left(a_{1}, \ldots, a_{n}\right)=$ $f\left(s^{-1}\left(a_{1}, \ldots, a_{n}\right)\right)$ (см. [1]).

Для произвольного класса функций $M \subset P_{2}(n)$ и группы $G<\operatorname{Sym}\left(V_{n}\right)$ обозначим через $G_{M}=\{g \in G: g(f)=f \forall f \in M\}$ поточечный стабилизатор множества $M$, а через $G_{\{M\}}=\{g \in G: g(f) \in M \forall f \in M\}$ - стабилизатор множества $M$. Ясно, что $G_{M}<G_{\{M\}}$.

В [1] доказан ряд фактов, которые во введенных обозначениях формулируются следующим образом:

1. Если $M \subset P_{2}(n)-$ класс функций, содержащий функции $\left\{x_{1}, \ldots, x_{n}\right\}$ $\left(\left\{\bar{x}_{1}, \ldots, \bar{x}_{n}\right\}\right)$ и $\sigma \in \operatorname{Sym}\left(V_{n}\right)_{\{M\}}$, то координатные функции $s_{1}, \ldots, s_{n}$ подстановки $\sigma$ лежат в $M$ (все инверсии $\bar{s}_{1}, \ldots, \bar{s}_{n}$ координатных функций $s_{1}, \ldots, s_{n}$ подстановки $\sigma$ лежат в $\left.M\right)$.

2. $\operatorname{Sym}\left(V_{n}\right)_{\{A(n)\}}=A G L_{n}$.

3. $\operatorname{Sym}\left(V_{n}\right)_{\{B i(n)\}}=Q_{n}$.

4. $\operatorname{Sym}\left(V_{n}\right)_{\{W P(n)\}}=\operatorname{Sym}\left(V_{n}\right)_{\{W N(n)\}}=\widetilde{S}_{n}$.

В дальнейшем для краткости группу $\operatorname{Sym}\left(V_{n}\right)$ будем обозначать через $\mathfrak{G}$. 


\section{2. Основные результаты}

В [1] и [2] описаны стабилизаторы в группе $\operatorname{Sym}\left(V_{n}\right)$ для некоторых классов функций от $n$ переменных из Галуа-замкнутых подклассов классов Шефера, а именно для первых 29 классов из таблицы 1 (в [2] описано 34 группы, но с учетом неточного результата для класса $M_{2}$ и равенств $B i_{1}=W P_{2,2}^{1}$, $B i_{0}=W N_{2,2}^{0}, \quad M_{4}=W P_{2,2} \quad$ и $M_{5}=W N_{2,2}$ получаем 29 классов). Для завершения описания исследуемых групп необходимо описать стабилизаторы множеств функций от $n$ переменных из классов с 30-го по 46-й из таблицы 1.

Рассмотрим класс $A^{01}=A \cap 0-S \cap 1-S$ и построим его стабилизатор $\mathfrak{G}_{\left\{A^{01}(n)\right\}}$ при $n \geqslant 3$.

Теорема 2. Функияия $f \in A^{01}(n)$ тогда и только тогда, когда $E_{f}$ - подпространство в $V_{n}$, содержащее вектор $1_{n}$.

Доказательство. Необходимость. Пусть $f \in A^{01}(n)$. Поскольку $A^{01} \subset A$, $E_{f}-$ линейное многообразие в $V_{n}$. Так как $A^{01} \subset 0-S$, то $0_{n} \in E_{f}$ и $E_{f}<V_{n}$. Поскольку $A^{01} \subset 1-S$, имеет место принадлежность $1_{n} \in E_{f}$.

Достаточность. Из того, что $E_{f}<V_{n}$, очевидно следует, что $f \in A^{0}$. Тогда, так как $1_{n} \in E_{f}$, то $f \in A^{01}$.

Очевидно включение $\Sigma_{n}<\mathfrak{G}_{\left\{A^{01}(n)\right\}}$, из которого следует, что группа $\mathfrak{G}_{\left\{A^{01}(n)\right\}}$ транзитивна.

Определим разбиение пространства $V_{n}$ на $2^{n-1}$ блоков:

$$
\mathbb{B}=\left\{\Delta_{\alpha^{\prime}}, \alpha^{\prime} \in V_{n-1}\right\}, \quad \text { где } \quad \Delta_{\alpha^{\prime}}=\left\{\left(\alpha^{\prime}, 0\right),\left(\bar{\alpha}^{\prime}, 1\right)\right\} .
$$

Теорема 3. При $n \geqslant 3$ группа $\mathfrak{G}_{\left\{A^{01}(n)\right\}}$ импримитивна с системой блоков импримитивности $\mathbb{B}$.

Доказательство. Если $g \in \mathfrak{G}_{\left\{A^{01}(n)\right\}}$, то подстановка $g$ переводит любое подпространство, содержащее $1_{n}$, в подпространство, содержащее $1_{n}$. При этом сохраняется размерность подпространства. Положим $L_{0}=\left\{0_{n}, 1_{n}\right\}$. Тогда $\operatorname{dim} L_{0}=1$. Других подпространств размерности 1 , удовлетворяющих условию теоремы 1 , нет. Следовательно, $g\left(L_{0}\right)=L_{0}$.

Рассмотрим подпространство размерности 2 , удовлетворяющее условию теоремы 1. Оно имеет вид $L_{0}=\left\{0_{n}, \alpha, \bar{\alpha}, 1_{n}\right\}$, где $\bar{\alpha}=\alpha \oplus 1_{n}, \alpha \in V_{n}$. Тогда очевидно, что если $g(\alpha)=\beta$, то $g(\bar{\alpha})=\bar{\beta}$. Это означает, что подстановка $g$ инвариантна относительно инверсии, что доказывает теорему. 
Следуя [9], определим понятие табличного сплетения групп подстановок. Пусть $G<\operatorname{Sym}\left(\Omega_{1}\right), \quad H<\operatorname{Sym}\left(\Omega_{2}\right)$. Сплетением групп $G$ и $H$ назовем группу подстановок $G$ ? $H<\operatorname{Sym}\left(\Omega_{1} \times \Omega_{2}\right)$, состоящую из подстановок $k=$ $(g, h)$, где $g \in G, h \in H^{\Omega_{1}}$ с действием вида:

$$
k(x, y)=(g(x), h(x)(y))=\left(x^{g}, y^{h(x)}\right), \quad x \in \Omega_{1}, \quad y \in \Omega_{2} .
$$

Данное равенство позволяет описать импримитивную группу как подгруппу сплетения групп подстановок, из которых первая группа действует на блоках, а вторая - на элементах внутри блоков.

Рассмотрим систему блоков $\mathbb{B}$. Пусть $\gamma=\left(a_{1}, \ldots, a_{n}\right) \in V_{n}$. Тогда легко видеть, что вектор $\gamma$ лежит в блоке $\Delta_{\alpha^{\prime}}, \alpha^{\prime}=\left(a_{1} \oplus a_{n}, \ldots, a_{n-1} \oplus a_{n}\right)$. То есть $\alpha^{\prime}=\left(a_{1}, \ldots, a_{n-1}\right)$, если $a_{n}=0$, и $\alpha^{\prime}=\left(\bar{a}_{1}, \ldots, \bar{a}_{n-1}\right)$, если $a_{n}=1$. При этом $\Delta_{\alpha^{\prime}}=\left\{\left(a_{1} \oplus a_{n}, \ldots, a_{n-1} \oplus a_{n}, 0\right),\left(a_{1} \oplus a_{n} \oplus 1, \ldots, a_{n-1} \oplus a_{n} \oplus 1,1\right)\right\}$. Ясно, что $\Delta_{\alpha^{\prime}}=\{\gamma, \bar{\gamma}\}$.

По теореме 3 импримитивная группа $\mathfrak{G}_{\left\{A^{01}(n)\right\}}$ изоморфно вкладывается в сплетение $\operatorname{Sym}\left(V_{n-1}\right) \prec \operatorname{Sym}(\{0,1\}) \cong S_{2^{n-1}}\left\{S_{2}\right.$. Определим действие этого сплетения на множестве $V_{n}=V_{n-1} \times V_{1}$. Пусть $k=(g, h)$, где $g \in \operatorname{Sym}\left(V_{n-1}\right), h: V_{n-1} \rightarrow \operatorname{Sym}\left(V_{1}\right)$.

Ввиду того что группа $\operatorname{Sym}\left(V_{1}\right)$ состоит из двух подстановок (тождественной и транспозиции), можно определить действие $y^{h(x)}, y \in\{0,1\}$, как $y^{h(x)}=y \oplus \widetilde{h}(x)$, где

$$
\widetilde{h}(x)= \begin{cases}0, & h(x)=\varepsilon, \\ 1, & h(x)=(0,1) .\end{cases}
$$

Тогда $k(\gamma)=\left(g\left(a_{1} \oplus a_{n}, \ldots, a_{n-1} \oplus a_{n}\right) \oplus 1_{n-1}\left(a_{n} \oplus \widetilde{h}\left(a_{1} \oplus a_{n}, \ldots, a_{n-1} \oplus a_{n}\right)\right)\right.$, $\left.a_{n} \oplus \widetilde{h}\left(a_{1} \oplus a_{n}, \ldots, a_{n-1} \oplus a_{n}\right)\right)$.

Без ограничения общности всякую подстановку $k$, лежащую в некоторой импримитивной группе $G$ с системой блоков $\mathbb{B}$, будем обозначать парой $k=$ $(g, h)$, где $g \in \operatorname{Sym}\left(V_{n-1}\right), h: V_{n-1} \rightarrow \operatorname{Sym}\left(V_{1}\right)$.

Теорема 4. Если $k=(g, h) \in \mathfrak{G}_{\left\{A^{01}(n)\right\}}$, то $g \in G L_{n-1}$. Инылии словами, $\mathfrak{G}_{\left\{A^{01}(n)\right\}}<G L_{n-1} 2 S_{2}$.

Доказательство. Покажем, что $g$ - линейная подстановка. Пусть $\alpha=\left(a_{1}, \ldots, a_{n}\right), \beta=\left(b_{1}, \ldots, b_{n}\right)$ и $k(\alpha)=\gamma, k(\beta)=\delta, k=(g, h) \in \mathfrak{G}_{\left\{A^{01}\right\}(n)}$.

Рассмотрим подпространство $L<V_{n}$, порожденное векторами $\alpha, \beta, 1_{n}$. По теореме 2 данное пространство является носителем некоторой функции $f \in A^{01}(n)$. Так как $k \in \mathfrak{G}_{\left\{A^{01}(n)\right\}}$, то $k(L)<V_{n}$ и $1_{n} \in k(L)$. 
Тогда

$$
\begin{aligned}
L & =\left\{0_{n}, \alpha, \beta, \bar{\alpha}, \bar{\beta}, \alpha \oplus \beta, \alpha \oplus \beta \oplus 1_{n}, 1_{n}\right\}, \\
k(L) & =\left\{0_{n}, \gamma, \delta, \bar{\gamma}, \bar{\delta}, \gamma \oplus \delta, \gamma \oplus \delta \oplus 1_{n}, 1_{n}\right\} .
\end{aligned}
$$

Следовательно,

$$
k(\alpha \oplus \beta) \in\left\{\gamma \oplus \delta, \gamma \oplus \delta \oplus 1_{n}\right\}=\left\{k(\alpha) \oplus k(\beta), k(\alpha) \oplus k(\beta) \oplus 1_{n}\right\} .
$$

Поэтому

$$
\begin{aligned}
k(\alpha \oplus \beta)= & \left(g\left(a_{1} \oplus a_{n} \oplus b_{1} \oplus b_{n}, \ldots, a_{n-1} \oplus a_{n} \oplus b_{n-1} \oplus b_{n}\right) \oplus\right. \\
& \oplus 1_{n-1}\left(a_{n} \oplus b_{n} \oplus \widetilde{h}\left(a_{1} \oplus a_{n} \oplus b_{1} \oplus b_{n}, \ldots, a_{n-1} \oplus a_{n} \oplus b_{n-1} \oplus b_{n}\right),\right. \\
& \left.a_{n} \oplus b_{n} \oplus \widetilde{h}\left(a_{1} \oplus a_{n} \oplus b_{1} \oplus b_{n}, \ldots, a_{n-1} \oplus a_{n} \oplus b_{n-1} \oplus b_{n}\right)\right) .
\end{aligned}
$$

Это, в частности, означает, что либо

$$
\begin{aligned}
& a_{n} \oplus b_{n} \oplus \widetilde{h}\left(a_{1} \oplus a_{n} \oplus b_{1} \oplus b_{n}, \ldots, a_{n-1} \oplus a_{n} \oplus b_{n-1} \oplus b_{n}\right)= \\
& =a_{n} \oplus \widetilde{h}\left(a_{1} \oplus a_{n}, \ldots, a_{n-1} \oplus a_{n}\right) \oplus b_{n} \oplus \widetilde{h}\left(b_{1} \oplus b_{n}, \ldots, b_{n-1} \oplus b_{n}\right),
\end{aligned}
$$

либо

$$
\begin{aligned}
& a_{n} \oplus b_{n} \oplus \widetilde{h}\left(a_{1} \oplus a_{n} \oplus b_{1} \oplus b_{n}, \ldots, a_{n-1} \oplus a_{n} \oplus b_{n-1} \oplus b_{n}\right)= \\
& =a_{n} \oplus \widetilde{h}\left(a_{1} \oplus a_{n}, \ldots, a_{n-1} \oplus a_{n}\right) \oplus b_{n} \oplus \widetilde{h}\left(b_{1} \oplus b_{n}, \ldots, b_{n-1} \oplus b_{n}\right) \oplus 1
\end{aligned}
$$

В обоих случаях

$$
\begin{aligned}
& g\left(a_{1} \oplus a_{n} \oplus b_{1} \oplus b_{n}, \ldots, a_{n-1} \oplus a_{n} \oplus b_{n-1} \oplus b_{n}\right)= \\
& =g\left(a_{1} \oplus a_{n}, \ldots, a_{n-1} \oplus a_{n}\right) \oplus g\left(b_{1} \oplus b_{n}, \ldots, b_{n-1} \oplus b_{n}\right),
\end{aligned}
$$

т. е. $g \in G L_{n-1}$. 
Теорема 5. Для класса $A^{01}(n)=A(n) \cap 0-S(n) \cap 1-S(n)$ верно соотномение

$$
\mathfrak{G}_{\left\{A^{01}(n)\right\}} \cong G L_{n-1} \prec S_{2} .
$$

Доказательство. Пусть $k=(g, h) \in G L_{n-1} \backslash S_{2}$. Покажем, что если $L=E_{f}, f \in A^{01}(n)$, то $k(L)<V_{n}, 1_{n} \in k(L)$. Из теоремы 2 следует, что $L<V_{n}, 1_{n} \in E_{f}$. В случаях, когда $\operatorname{dim} L=1$ или $\operatorname{dim} L=2$, доказательство теоремы очевидным образом следует из импримитивности группы $G L_{n-1} 2 S_{2}$ с системой блоков $\mathbb{B}$.

Рассмотрим случай, когда $\operatorname{dim} L \geqslant 3$. Пусть $\alpha, \beta \in L, \gamma=k(\alpha), \delta=k(\beta)$, $\alpha=\left(a_{1}, \ldots, a_{n}\right), \beta=\left(b_{1}, \ldots, b_{n}\right)$. Введем векторы $\alpha^{\prime}=\left(a_{1} \oplus a_{n}, \ldots, a_{n-1} \oplus a_{n}\right)$, $\beta^{\prime}=\left(b_{1} \oplus b_{n}, \ldots, b_{n-1} \oplus b_{n}\right)$. Покажем, что $\gamma \oplus \delta \in k(L)$. Для этого рассмотрим подпространство

$$
L_{1}=\left\{0_{n}, \alpha, \beta, \bar{\alpha}, \bar{\beta}, \alpha \oplus \beta, \alpha \oplus \beta \oplus 1_{n}, 1_{n}\right\}<L .
$$

Имеем $k\left(L_{1}\right)=\left\{0_{n}, \gamma, \delta, \bar{\gamma}, \bar{\delta}, k(\alpha \oplus \beta), k(\alpha \oplus \beta) \oplus 1_{n}, 1_{n}\right\} \subset k(L)$, причем из свойств подстановки $k \in G L_{n-1} 2 S_{2}$ вытекают соотношения

$$
k(\bar{\alpha})=\bar{\gamma}, k(\bar{\beta})=\bar{\delta}, k\left(\left\{0_{n}, 1_{n}\right\}\right)=\left\{0_{n}, 1_{n}\right\} .
$$

Далее,

$$
\begin{aligned}
k(\alpha \oplus \beta)= & \left(g\left(\alpha^{\prime} \oplus \beta^{\prime}\right) \oplus 1_{n-1}\left(a_{n} \oplus b_{n} \oplus \widetilde{h}\left(\alpha^{\prime} \oplus \beta^{\prime}\right)\right), a_{n} \oplus b_{n} \oplus \widetilde{h}\left(\alpha^{\prime} \oplus \beta^{\prime}\right)\right)= \\
= & \left(g\left(\alpha^{\prime}\right) \oplus 1_{n-1}\left(a_{n} \oplus \widetilde{h}\left(\alpha^{\prime}\right)\right), a_{n} \oplus \widetilde{h}\left(\alpha^{\prime}\right)\right) \oplus \\
& \oplus\left(g\left(\beta^{\prime}\right) \oplus 1_{n-1}\left(b_{n} \oplus \widetilde{h}\left(\beta^{\prime}\right)\right), b_{n} \oplus \widetilde{h}\left(\beta^{\prime}\right)\right) \oplus \\
& \oplus\left(1_{n-1}\left(\widetilde{h}\left(\alpha^{\prime}\right) \oplus \widetilde{h}\left(\beta^{\prime}\right) \oplus \widetilde{h}\left(\alpha^{\prime} \oplus \beta^{\prime}\right)\right), \widetilde{h}\left(\alpha^{\prime}\right) \oplus \widetilde{h}\left(\beta^{\prime}\right) \oplus \widetilde{h}\left(\alpha^{\prime} \oplus \beta^{\prime}\right)\right)= \\
= & k(\alpha) \oplus k(\beta) \oplus \xi,
\end{aligned}
$$

где $\xi=\left(1_{n-1}\left(\widetilde{h}\left(\alpha^{\prime}\right) \oplus \widetilde{h}\left(\beta^{\prime}\right) \oplus \widetilde{h}\left(\alpha^{\prime} \oplus \beta^{\prime}\right)\right), \widetilde{h}\left(\alpha^{\prime}\right) \oplus \widetilde{h}\left(\beta^{\prime}\right) \oplus \widetilde{h}\left(\alpha^{\prime} \oplus \beta^{\prime}\right)\right) \in\left\{0_{n}, 1_{n}\right\}$.

Поэтому $k(\alpha \oplus \beta) \in\left\{\gamma \oplus \delta, \gamma \oplus \delta \oplus 1_{n}\right\}$. Аналогично

$$
k\left(\alpha \oplus \beta \oplus 1_{n}\right) \in\left\{\gamma \oplus \delta, \gamma \oplus \delta \oplus 1_{n}\right\} .
$$

Это означает, что

$$
k\left(\left\{\alpha \oplus \beta, \alpha \oplus \beta \oplus 1_{n}\right\}\right)=\left\{\gamma \oplus \delta, \gamma \oplus \delta \oplus 1_{n}\right\} .
$$

Следовательно, $\gamma \oplus \delta, \gamma \oplus \delta \oplus 1_{n} \in k\left(L_{1}\right) \subset k(L)$. Отсюда $k(L)<V_{n}, 1_{n} \in k(L)$. Значит, $k \in G_{\left\{A^{01}(n)\right\}}$. 
Теорема 6. Для класса $M_{8}(n)=A(n) \cap C L(n)$ верно соотношение

$$
\mathfrak{G}_{\left\{M_{8}(n)\right\}} \cong A G L_{n-1}\left\langle S_{2} .\right.
$$

Доказательство. Доказательство этой теоремы строится аналогично доказательству теоремы 5. Сначала заметим, что носителями функций $f\left(x_{1}, \ldots, x_{n}\right) \in M_{8}(n)$ являются линейные многообразия вида $\xi \oplus E_{p}$, где $\xi \in V_{n}, p\left(x_{1}, \ldots, x_{n}\right) \in A^{01}(n)$. Это все многообразия, содержащие векторы вместе с их инверсиями. Поэтому группа $\mathfrak{G}_{\left\{M_{8}(n)\right\}}$ импримитивна с системой блоков $\mathbb{B}$. Следовательно, группа $\mathfrak{G}_{\left\{M_{8}(n)\right\}}$ изоморфно вкладывается в сплетение $\operatorname{Sym}\left(V_{n-1}\right)$ < $S_{2}$.

Рассмотрим многообразие вида $L=E_{f}, f \in M_{8}(n)$, размерности 3. Выпишем векторы из данного многообразия в виде

$$
L=\left\{\alpha, \beta, \gamma, \bar{\alpha}, \bar{\beta}, \bar{\gamma}, \alpha \oplus \beta \oplus \gamma, \alpha \oplus \beta \oplus \gamma \oplus 1_{n}\right\} .
$$

Обратно, очевидно, что всякое многообразие такого вида является носителем некоторой функции $f\left(x_{1}, \ldots, x_{n}\right) \in M_{8}(n)$. Покажем, что если $k=(g, h) \in \mathfrak{G}_{\left\{M_{8}(n)\right\}}$, то $g \in A G L_{n-1}$. Для этого достаточно показать, что $g(\alpha \oplus \beta \oplus \gamma)=g(\alpha) \oplus g(\beta) \oplus g(\gamma)$. Пусть $k(\alpha)=\alpha_{1}, k(\beta)=\beta_{1}, k(\gamma)=\gamma_{1}$.

Тогда $k(L)=\left\{\alpha_{1}, \beta_{1}, \gamma_{1}, \bar{\alpha}_{1}, \bar{\beta}_{1}, \overline{\gamma_{1}}, \alpha_{1} \oplus \beta_{1} \oplus \gamma_{1}, \alpha_{1} \oplus \beta_{1} \oplus \gamma_{1} \oplus 1_{n}\right\}$.

Из импримитивности группы $\mathfrak{G}_{\left\{A^{01}(n)\right\}}$ с системой блоков $\mathbb{B}$ следует, что $k(\bar{\alpha})=\bar{\alpha}_{1}, k(\bar{\beta})=\bar{\beta}_{1}, k(\bar{\gamma})=\bar{\gamma}_{1}$.

Следовательно, $k(\alpha \oplus \beta \oplus \gamma) \in\left\{\alpha_{1} \oplus \beta_{1} \oplus \gamma_{1}, \alpha_{1} \oplus \beta_{1} \oplus \gamma_{1} \oplus 1_{n}\right\}=$ $=\left\{k(\alpha) \oplus k(\beta) \oplus k(\gamma), k(\alpha) \oplus k(\beta) \oplus k(\gamma) \oplus 1_{n}\right\}$.

Пусть $\alpha=\left(a_{1}, \ldots, a_{n}\right), \beta=\left(b_{1}, \ldots, b_{n}\right), \gamma=\left(c_{1}, \ldots, c_{n}\right)$. Тогда

$k(\alpha \oplus \beta \oplus \gamma)=\left(g\left(a_{1} \oplus a_{n} \oplus b_{1} \oplus b_{n} \oplus c_{1} \oplus c_{n}, \ldots\right.\right.$

$$
\begin{gathered}
\left.\ldots, a_{n-1} \oplus a_{n} \oplus b_{n-1} \oplus b_{n} \oplus c_{n-1} \oplus c_{n}\right) \oplus 1_{n-1} \oplus \\
\oplus\left(a _ { n } \oplus b _ { n } \oplus c _ { n } \oplus \widetilde { h } \left(a_{1} \oplus a_{n} \oplus b_{1} \oplus b_{n} \oplus c_{1} \oplus c_{n}, \ldots\right.\right. \\
\left.\left.\ldots, a_{n-1} \oplus a_{n} \oplus b_{n-1} \oplus b_{n} \oplus c_{n-1} \oplus c_{n}\right)\right), \\
a_{n} \oplus b_{n} \oplus c_{n} \oplus \widetilde{h}\left(a_{1} \oplus a_{n} \oplus b_{1} \oplus b_{n} \oplus c_{1} \oplus c_{n}, \ldots\right. \\
\left.\left.\ldots, a_{n-1} \oplus a_{n} \oplus b_{n-1} \oplus b_{n} \oplus c_{n-1} \oplus c_{n}\right)\right) .
\end{gathered}
$$

Это, в частности, означает, что либо $a_{n} \oplus b_{n} \oplus c_{n} \oplus \widetilde{h}\left(a_{1} \oplus a_{n} \oplus b_{1} \oplus b_{n} \oplus c_{1} \oplus c_{n}\right.$, $\left.\ldots, a_{n-1} \oplus a_{n} \oplus b_{n-1} \oplus b_{n} \oplus c_{n-1} \oplus c_{n}\right)=a_{n} \oplus \widetilde{h}\left(a_{1} \oplus a_{n}, \ldots, a_{n-1} \oplus a_{n}\right) \oplus b_{n} \oplus$ $\underset{\sim}{\widetilde{h}}\left(b_{1} \oplus b_{n}, \ldots, b_{n-1} \oplus b_{n}\right) \oplus c_{n} \oplus \widetilde{h}\left(c_{1} \oplus c_{n}, \ldots, c_{n-1} \oplus c_{n}\right)$, либо $a_{n} \oplus b_{n} \oplus$ $\widetilde{h}\left(a_{1} \oplus a_{n} \oplus b_{1} \oplus b_{n} \oplus c_{1} \oplus c_{n}, \ldots, a_{n-1} \oplus a_{n} \oplus b_{n-1} \oplus b_{n} \oplus c_{n-1} \oplus c_{n}\right)=$ $=a_{n} \oplus \widetilde{h}\left(a_{1} \oplus a_{n}, \ldots, a_{n-1} \oplus a_{n}\right) \oplus b_{n} \oplus \widetilde{h}\left(b_{1} \oplus b_{n}, \ldots, b_{n-1} \oplus b_{n}\right) \oplus c_{n} \oplus$ $\oplus \widetilde{h}\left(c_{1} \oplus c_{n}, \ldots, c_{n-1} \oplus c_{n}\right) \oplus 1$. 
В обоих случаях

$g\left(a_{1} \oplus a_{n} \oplus b_{1} \oplus b_{n} \oplus c_{1} \oplus c_{n}, \ldots, a_{n-1} \oplus a_{n} \oplus b_{n-1} \oplus b_{n} \oplus \oplus c_{n-1} \oplus c_{n}\right)=$ $=g\left(a_{1} \oplus a_{n}, \ldots, a_{n-1} \oplus a_{n}\right) \oplus g\left(b_{1} \oplus b_{n}, \ldots, b_{n-1} \oplus b_{n}\right) \oplus g\left(c_{1} \oplus c_{n}, \ldots, c_{n-1} \oplus c_{n}\right)$,

откуда следует, что $g \in A G L_{n-1}$. Таким образом, $\mathfrak{G}_{\left\{M_{8}(n)\right\}} \subset A G L_{n-1} \prec S_{2}$. Покажем, что имеет место обратное включение.

Пусть $k=(g, h) \in A G L_{n-1} \curlywedge S_{2}$. Покажем, что если $L=E_{f}, f \in M_{8}(n)$, то $k(L)$ - линейное многообразие, содержащее векторы вместе с их отрицаниями. Если $\operatorname{dim} L=1$, то многообразие $L$ имеет вид $L=\{\alpha, \bar{\alpha}\}$, т. е. является блоком из системы блоков $\mathbb{B}$. В этом случае теорема, очевидно, верна. Если же $\operatorname{dim} L=2$, то $L=\{\alpha, \bar{\alpha}, \beta, \bar{\beta}\}$. Любое такое многообразие является носителем некоторой функции из $M_{8}$. Поэтому при действии подстановки $k$ оно перейдет в аналогичное многообразие, и теорема верна.

Рассмотрим случай, когда $\operatorname{dim} L \geqslant 3$. Пусть $\alpha, \beta, \gamma \in L, \alpha_{1}=k(\alpha)$, $\beta_{1}=k(\beta), \gamma_{1}=k(\gamma), \alpha=\left(a_{1}, \ldots, a_{n}\right), \beta=\left(b_{1}, \ldots, b_{n}\right), \gamma=\left(c_{1}, \ldots, c_{n}\right)$. Введем векторы $\alpha^{\prime}=\left(a_{1} \oplus a_{n}, \ldots, a_{n-1} \oplus a_{n}\right), \beta^{\prime}=\left(b_{1} \oplus b_{n}, \ldots, b_{n-1} \oplus b_{n}\right)$, $\gamma^{\prime}=\left(c_{1} \oplus c_{n}, \ldots, c_{n-1} \oplus c_{n}\right)$.

Покажем, что $\alpha_{1} \oplus \beta_{1} \oplus \gamma_{1} \in k(L)$. Для этого рассмотрим многообразие

$$
L_{1}=\left\{\alpha, \beta, \gamma, \bar{\alpha}, \bar{\beta}, \bar{\gamma}, \alpha \oplus \beta \oplus \gamma, \alpha \oplus \beta \oplus \gamma \oplus 1_{n}\right\} .
$$

Имеем

$$
k\left(L_{1}\right)=\left\{\alpha_{1}, \beta_{1}, \gamma_{1}, \bar{\alpha}_{1}, \bar{\beta}_{1}, \bar{\gamma}_{1}, k(\alpha \oplus \beta \oplus \gamma), k\left(\alpha \oplus \beta \oplus \gamma \oplus 1_{n}\right)\right\} \subset k(L),
$$

причем из импримитивности группы $A G L_{n-1} 2 S_{2}$ вытекают соотношения $k(\bar{\alpha})=\bar{\alpha}_{1}, k(\bar{\beta})=\bar{\beta}_{1}, k(\bar{\gamma})=\bar{\gamma}_{1}, k\left(\alpha \oplus \beta \oplus \gamma \oplus 1_{n}\right)=k(\alpha \oplus \beta \oplus \gamma) \oplus 1_{n}$.

Далее,

$$
\begin{aligned}
k(\alpha \oplus \beta \oplus \gamma)= & \left(g\left(\alpha^{\prime} \oplus \beta^{\prime} \oplus \gamma^{\prime}\right) \oplus 1_{n-1}\left(a_{n} \oplus b_{n} \oplus c_{n} \oplus \widetilde{h}\left(\alpha^{\prime} \oplus \beta^{\prime} \oplus \gamma^{\prime}\right)\right),\right. \\
& \left.a_{n} \oplus b_{n} \oplus c_{n} \oplus \widetilde{h}\left(\alpha^{\prime} \oplus \beta \oplus \gamma^{\prime \prime}\right)\right)= \\
= & \left(g\left(\alpha^{\prime}\right) \oplus 1_{n-1}\left(a_{n} \oplus \widetilde{h}\left(\alpha^{\prime}\right)\right), a_{n} \oplus \widetilde{h}\left(\alpha^{\prime}\right)\right) \oplus \\
& \oplus\left(g\left(\beta^{\prime}\right) \oplus 1_{n-1}\left(b_{n} \oplus \widetilde{h}\left(\beta^{\prime}\right)\right), b_{n} \oplus \widetilde{h}\left(\beta^{\prime}\right)\right) \oplus \\
& \left(g\left(\gamma^{\prime}\right) \oplus 1_{n-1}\left(c_{n} \oplus \widetilde{h}\left(\gamma^{\prime}\right)\right), c_{n} \oplus \widetilde{h}\left(\gamma^{\prime}\right)\right) \oplus \\
\oplus & \left(1_{n}\left(\widetilde{h}\left(\alpha^{\prime}\right) \oplus \widetilde{h}\left(\beta^{\prime}\right) \oplus \widetilde{h}\left(\gamma^{\prime}\right) \oplus \widetilde{h}\left(\alpha^{\prime} \oplus \beta^{\prime} \oplus \gamma^{\prime}\right)\right),\right. \\
& \left.\widetilde{h}\left(\alpha^{\prime}\right) \oplus \widetilde{h}\left(\beta^{\prime}\right) \oplus \widetilde{h}\left(\gamma^{\prime}\right) \oplus \widetilde{h}\left(\alpha^{\prime} \oplus \beta^{\prime} \oplus \gamma^{\prime}\right)\right) \\
= & k(\alpha) \oplus k(\beta) \oplus \xi,
\end{aligned}
$$

где $\xi=1_{n}\left(\widetilde{h}\left(\alpha^{\prime}\right) \oplus \widetilde{h}\left(\beta^{\prime}\right) \oplus \widetilde{h}\left(\gamma^{\prime}\right) \oplus \widetilde{h}\left(\alpha^{\prime} \oplus \beta^{\prime} \oplus \gamma^{\prime}\right)\right) \in\left\{0_{n}, 1_{n}\right\}$. 
Поэтому $k(\alpha \oplus \beta \oplus \gamma) \in\left\{\alpha_{1} \oplus \beta_{1} \oplus \gamma_{1}, \alpha_{1} \oplus \beta_{1} \oplus \gamma_{1} \oplus 1_{n}\right\}$. Аналогично

$$
k\left(\alpha \oplus \beta \oplus \gamma \oplus 1_{n}\right) \in\left\{\alpha_{1} \oplus \beta_{1} \oplus \gamma_{1}, \alpha_{1} \oplus \beta_{1} \oplus \gamma_{1} \oplus 1_{n}\right\} .
$$

Это означает, что

$$
k\left(\left\{\alpha \oplus \beta \oplus \gamma, \alpha \oplus \beta \oplus \gamma \oplus 1_{n}\right\}\right)=\left\{\alpha_{1} \oplus \beta_{1} \oplus \gamma_{1}, \alpha_{1} \oplus \beta_{1} \oplus \gamma_{1} \oplus 1_{n}\right\} .
$$

Следовательно, $\alpha_{1} \oplus \beta_{1} \oplus \gamma_{1}, \alpha_{1} \oplus \beta_{1} \oplus \gamma_{1} \oplus 1_{n} \in k\left(L_{1}\right) \subset k(L)$. Таким образом, $k(L)$ - многообразие, содержащее инверсии своих векторов. Значит, $k \in \mathfrak{G}_{\left\{M_{8}(n)\right\}}$.

Перейдем к описанию групп $\mathfrak{G}_{\left\{M_{2}(n)\right\}}$ и $\mathfrak{G}_{\left\{M_{7}(n)\right\}}$. Следующая теорема уточняет теорему 29 из [2].

Теорема 7. При $n \geqslant 3$ для класса $M_{2}(n)=B i(n) \cap A(n)$ верно соотношение

$$
\mathfrak{G}_{\left\{M_{2}(n)\right\}}=A R_{n}^{(1)} .
$$

Доказательство. Сначала покажем, что $A R_{n}^{(1)}<\mathfrak{G}_{\left\{M_{2}(n)\right\}}$. Очевидно, что $Q_{n}<\mathfrak{G}_{\left\{M_{2}(n)\right\}}$. Покажем, что класс $M_{2}(n)$ инвариантен относительно невырожденного линейного преобразования пространства $V_{n}$, задаваемого матрицей $T_{n}$. Это преобразование $\tau_{n}$ имеет следующий вид: если $\alpha=$ $\left(a_{1}, a_{2}, \ldots, a_{n-1}, a_{n}\right) \in V_{n}$, то

$$
\tau_{n}(\alpha)=\alpha \cdot T_{n}=\left(a_{1} \oplus a_{n}, a_{2} \oplus a_{n}, \ldots, a_{n-1} \oplus a_{n}, a_{n}\right) .
$$

Для произвольного множества $W \subset V_{n}$ введем обозначения $W \cdot T_{n}=$ $\left\{\alpha \cdot T_{n} \mid \alpha \in W\right\}$ и $f_{W}$ - функция-индикатор множества $W$. Для произвольных $i, j \in \overline{1, n}, a \in\{0,1\}$ легко показать, что при $W \in\left\{E_{x_{i}}, E_{\bar{x}_{i}}, E_{x_{i} \oplus x_{j} \oplus a}\right\}$ выполнено $f_{W \cdot T_{n}} \in M_{2}(n)$.

Следовательно, $\tau_{n} \in \mathfrak{G}_{\left\{M_{2}(n)\right\}}$, а тогда $R_{n}^{(1)}<\mathfrak{G}_{\left\{M_{2}(n)\right\}}$. Так как $\Sigma_{n}<Q_{n}$ и $A R_{n}^{(1)}=\left\langle R_{n}^{(1)}, \Sigma_{n}\right\rangle$, то $A R_{n}^{(1)}<\mathfrak{G}_{\left\{M_{2}(n)\right\}}$.

Докажем, что имеет место обратное включение. Пусть $\sigma=\left(s_{1}, \ldots, s_{n}\right) \in$ $\mathfrak{G}_{\left\{M_{2}(n)\right\}}$. Поскольку функции $x_{1}, \ldots, x_{n}, \bar{x}_{1}, \ldots, \bar{x}_{n}$ лежат в $M_{2}(n)$, по лемме 2 из [1] (см. факт 1 в конце первого раздела настоящей статьи) $s_{1}, \ldots, s_{n}$ - равновероятные функции из $M_{2}(n)$. Однако легко видеть, что множество равновероятных мультиаффинных биюнктивных функций исчерпывается функциями $x_{1}, \ldots, x_{n}, \bar{x}_{1}, \ldots, \bar{x}_{n}$ и функциями вида $x_{i} \oplus x_{j} \oplus a$, где $i, j \in \overline{1, n}, a \in\{0,1\}$. Поэтому преобразование $\sigma$ является подстановкой из $A G L_{n}$, причем матрица этого преобразования состоит из столбцов веса не более 2 . 
Без ограничения общности (с точностью до преобразования из группы $\Sigma_{n}$ ) будем считать, что $\sigma(\alpha)=\alpha \cdot C$ для $\alpha=\left(a_{1}, a_{2}, \ldots, a_{n-1}, a_{n}\right) \in V_{n}$, где $C=\left(c_{i j}\right)_{n \times n}-$ невырожденная $n \times n$-матрица, столбцы которой имеют вес не более 2 . В силу включения $\widetilde{S}_{n}<Q_{n}<\mathfrak{G}_{\left\{M_{2}(n)\right\}}$ очевидно, что любая матрица, получаемая из матрицы $C$ перестановкой строк или столбцов, также задает преобразование из группы $\mathfrak{G}_{\left\{M_{2}(n)\right\}}$. Если все столбцы матрицы $C$ имеют вес 1 , то $C-$ подстановочная матрица и $\sigma \in \widetilde{S}_{n}$. Пусть в матрице $C$ есть столбец веса 2. Поскольку матрица $C$ - невырожденная, в ней есть столбец веса 1 (иначе все столбцы - веса 2, сумма строк равна $0_{n}$ и матрица вырождена). Без ограничения общности будем считать, что этот столбец имеет номер $n$ и единица в нем стоит на $n$-м месте: $C_{n}^{\downarrow}=\left(\begin{array}{c}0 \\ \ldots \\ 0 \\ 1\end{array}\right)$. Покажем, что $\vec{C}_{n}=1_{n}$. С точностью до перестановки столбцов матрицы $C$ будем считать, что столбец веса 2 имеет номер 1. В этом случае либо (с точностью до перестановки первых $n-1$ строк) $C_{1}^{\downarrow}=\left(\begin{array}{c}1 \\ 1 \\ 0 \\ \cdots \\ 0 \\ 0\end{array}\right)$, либо $C_{1}^{\downarrow}=\left(\begin{array}{c}1 \\ 0 \\ \ldots \\ 0 \\ 1\end{array}\right)$.

Рассмотрим первый случай. Положим $f=x_{1} \oplus x_{n}$. Тогда $f(x C)=x_{1} \oplus$ $x_{2} \oplus x_{n} \notin M_{2}(n)$. Следовательно, первый случай невозможен.

Значит, имеет место второй случай. Покажем, что все столбцы, кроме $n$-го, имеют вес 2.

Предположим противное. Без ограничения общности считаем, что вес 2-го столбца равен 1 и он имеет вид $C_{2}^{\downarrow}=\left(\begin{array}{c}0 \\ 1 \\ \cdots \\ 0 \\ 0\end{array}\right)$ (на первом и последнем местах в столбце единица стоять не может, поскольку тогда матрица $C$ окажется вырожденной). Значит, верно соотношение $E_{x_{1} \oplus x_{2}} \cdot C=E_{x_{1} \oplus x_{2} \oplus x_{n}}$. Но $x_{1} \oplus$ $x_{2} \oplus x_{n} \notin M_{2}(n)$, следовательно, предположение неверно. Поэтому веса всех столбцов с 1 по $(n-1)$-й равны 2. Покажем, что $n$-й элемент каждого столбца равен 1. Рассмотрим столбец $C_{2}^{\downarrow}$. Аналогично проведенным рассуждениям считаем, что элемент $c_{2,2}=1$. Тогда $c_{i, 2}=1$ для некоторого $i \in\{1\} \cup \overline{3, n}$.

Рассмотрим функцию $f=x_{2} \oplus x_{n}$. Тогда $f(x C)=x_{2} \oplus x_{i} \oplus x_{n}$ и эта функция существенно зависит не более чем от 2 переменных. Значит, $i=n$. Для других столбцов доказательство строится аналогично. Таким образом, $\mathfrak{G}_{\left\{M_{2}(n)\right\}}=A R_{n}^{(1)}$. 
Рассмотрим группу $\mathfrak{G}_{\left\{M_{7}(n)\right\}}$ · Данная группа, очевидно, содержит группу $Q_{n}$, а значит, является транзитивной. Поскольку

$$
M_{7}(n)=B i(n) \cap C L(n) \subset C L(n),
$$

как и в случае с группой $\mathfrak{G}_{\left\{A^{01}\right\}}$, группа $\mathfrak{G}_{\left\{M_{7}(n)\right\}}$ является импримитивной с системой блоков $\mathbb{B}$. В этом случае носитель каждой функции из данного класса суть объединение некоторого количества таких блоков.

Пусть $f\left(x_{1}, \ldots, x_{n}\right) \in C L(n)$ и $E_{f}=\Delta_{\alpha_{1}^{\prime}} \cup \cdots \cup \Delta_{\alpha_{m}^{\prime}}$. Поставим функции $f$ в соответствие такую функцию $\widetilde{f} \in P_{2}(n-1)$, что $E_{\widetilde{f}}=\left\{\alpha_{1}^{\prime}, \ldots, \alpha_{m}^{\prime}\right\}$. Это соответствие, очевидно, является взаимно однозначным соответствием множеств $C L(n)$ и $P_{2}(n-1)$.

Лемма 8. При $n \geqslant 3$ функциия $f \in M_{7}(n)$ тогда и только тогда, когда $\widetilde{f} \in M_{2}(n-1)$.

Доказательство. Пусть $E_{f}=\Delta_{\alpha_{1}^{\prime}} \cup \cdots \cup \Delta_{\alpha_{m}^{\prime}}$ и $E_{\widetilde{f}}=\left\{\alpha_{1}^{\prime}, \ldots, \alpha_{m}^{\prime}\right\}$. Предположим, что выполнено первое включение. Тогда, поскольку $f \in B i(n)$, по критерию биюнктивности (теорема 1) для любых $i, j, k \in \overline{1, m}$ найдется такое $l \in \overline{1, m}$, что $v_{n}\left(\left(\alpha_{i}^{\prime}, 0\right),\left(\alpha_{j}^{\prime}, 0\right),\left(\alpha_{k}^{\prime}, 0\right)\right)=\left(\alpha_{l}^{\prime}, 0\right)$, где $v_{n}(\alpha, \beta, \gamma)=\alpha \beta \oplus \alpha \gamma \oplus$ $\beta \gamma-$ покоординатная операция. Но тогда $\alpha_{l}^{\prime} \in E_{\widetilde{f}}$ и $\alpha_{l}^{\prime}=v_{n-1}\left(\alpha_{i}^{\prime}, \alpha_{j}^{\prime}, \alpha_{k}^{\prime}\right)$. Таким образом, $\tilde{f} \in B i(n-1)$. Заметим, что класс $M_{7}(n)$ является подклассом в классе функций $A(n)$. Следовательно, для множества $E_{f}$ выполняется критерий мультиаффинности, т. е. для любых $i, j, k \in \overline{1, m}$ найдется такое $t \in \overline{1, m}$, что $\left(\alpha_{i}^{\prime}, 0\right) \oplus\left(\alpha_{j}^{\prime}, 0\right) \oplus\left(\alpha_{k}^{\prime}, 0\right)=\left(\alpha_{t}^{\prime}, 0\right)$. Значит, $\alpha_{i}^{\prime} \oplus \alpha_{j}^{\prime} \oplus \alpha_{k}^{\prime}=\alpha_{t}^{\prime}$ и $\tilde{f} \in A(n-1)$.

Пусть теперь $\widetilde{f} \in M_{2}(n-1)$. Построим соответствующую функцию $f \in$ $C L(n)$ и покажем, что $f \in M_{7}(n)$. Рассмотрим векторы $\alpha_{i}^{\prime}, \alpha_{j}^{\prime}, \alpha_{k}^{\prime} \in E_{\tilde{f}}$. Воспользуемся критериями мультиаффинности и биюнктивности. Обозначим

$$
v_{n-1}\left(\alpha_{i}^{\prime}, \alpha_{j}^{\prime}, \alpha_{k}^{\prime}\right)=\alpha_{l}^{\prime} \in E_{\widetilde{f}} \quad \text { и } \quad \alpha_{i}^{\prime} \oplus \alpha_{j}^{\prime} \oplus \alpha_{k}^{\prime}=\alpha_{t}^{\prime} \in E_{\widetilde{f}} .
$$

Тогда множество $E_{f}$ содержит векторы $\left(\alpha_{i}^{\prime}, 0\right),\left(\overline{\alpha_{i}^{\prime}}, 1\right),\left(\alpha_{j}^{\prime}, 0\right)\left(\overline{\alpha_{j}^{\prime}}, 1\right),\left(\alpha_{k}^{\prime}, 0\right)$, $\left(\overline{\alpha_{k}^{\prime}}, 1\right),\left(\alpha_{l}^{\prime}, 0\right),\left(\overline{\alpha_{l}^{\prime}}, 1\right),\left(\alpha_{t}^{\prime}, 0\right),\left(\overline{\alpha_{t}^{\prime}}, 1\right)$.

Пусть $\alpha=\left(\alpha_{i}^{\prime}, 0\right) \oplus a \cdot 1_{n}, \beta=\left(\alpha_{j}^{\prime}, 0\right) \oplus b \cdot 1_{n}, \gamma=\left(\alpha_{k}^{\prime}, 0\right) \oplus c \cdot 1_{n}, a, b, c \in\{0,1\}$. Тогда очевидно, что $\alpha \oplus \beta \oplus \gamma \in\left\{\left(\alpha_{t}^{\prime}, 0\right),\left(\overline{\alpha_{t}^{\prime}}, 1\right)\right\}$. Следовательно, $f \in A(n)$. Дальнейшая часть доказательства вытекает из соотношений

$$
\begin{aligned}
& v_{n}\left(\left(\alpha_{i}^{\prime}, 0\right),\left(\alpha_{j}^{\prime}, 0\right),\left(\alpha_{k}^{\prime}, 0\right)\right)=\left(\alpha_{l}^{\prime}, 0\right), \\
& v_{n}\left(\left(\alpha_{i}^{\prime}, 0\right),\left(\alpha_{j}^{\prime}, 0\right),\left(\overline{\alpha^{\prime}} k, 1\right)\right)=\left(\alpha_{l}^{\prime}, 0\right) \oplus\left(\alpha_{i}^{\prime}, 0\right) \oplus\left(\alpha_{j}^{\prime}, 0\right) \in E_{f}, \\
& v_{n}\left(\left(\alpha_{i}^{\prime}, 0\right),\left(\alpha_{j}^{\prime}, 0\right),\left(\overline{\alpha^{\prime}} k, 1\right)\right)=\left(\alpha_{l}^{\prime}, 0\right) \oplus\left(\alpha_{j}^{\prime}, 0\right) \oplus\left(\alpha_{k}^{\prime}, 0\right) \in E_{f} .
\end{aligned}
$$


Рассмотрим группу $H_{n}=\left\langle(\alpha, \bar{\alpha}), \alpha \in V_{n}\right\rangle$. Очевидно, что $\left|H_{n}\right|=2^{2^{n-1}}$ и $Q_{n} \cap H_{n}=\left\{\varepsilon, \widetilde{1}_{n}\right\}$. Легко показать, что группа $H_{n}$ изоморфна табличному сплетению групп $E\left(V_{n-1}\right)$ \ $S_{2}$, где $E\left(V_{n-1}\right)$ - группа, состоящая из одной тождественной подстановки на множестве $V_{n-1}$. Положим $Y_{n}=\left\langle Q_{n}, H_{n}\right\rangle$.

Лемма 9. При $n \geqslant 3$ верны соотношения

$$
H_{n} \triangleleft Y_{n}, \quad\left|Y_{n}\right|=2^{2^{n-1}+n-1} \cdot n !
$$

Доказательство. Для доказательства первого включения достаточно показать, что для $\sigma \in Q_{n}, \tau \in H_{n}$ выполнено $\sigma \tau \sigma^{-1} \in H_{n}$. Рассмотрим действие подстановки $\sigma$ на блоки системы $\mathbb{B}$. В силу импримитивности группы $Q_{n}$ данная подстановка сохраняет разбиение на блоки, переводя блок с номером $(0, \ldots, 0)$ в блок с номером $\beta_{0}, \ldots$, блок с номером $(1, \ldots, 1)-$ в блок с номером $\beta_{2^{n-1}-1}$. Подстановка $\tau$ стабилизирует блоки (сохраняет их на месте), а подстановка $\sigma^{-1}$ совершает обратное преобразование блоков. Поэтому подстановка $\sigma \tau \sigma^{-1}$ импримитивно действует на блоках, а значит, $\sigma \tau \sigma^{-1} \in H_{n}$ и $H_{n} \triangleleft Y_{n}$.

Докажем второе равенство. Очевидно, что $\left|Y_{n}\right| \leqslant\left|Q_{n}\right| \cdot\left|H_{n}\right|=2^{2^{n-1}+n} \cdot n$ ! Далее, в силу нормальности группы $H_{n}$ в $Y_{n}$ каждая подстановка $\sigma$ из $Y_{n}$ может быть представлена в виде

$$
\sigma=\pi \cdot \eta, \quad \pi \in Q_{n}, \eta \in H_{n}
$$

Положим теперь $\pi^{\prime}(x)=\pi(x) \oplus 1_{n}, \eta^{\prime}(x)=\eta(x) \oplus 1_{n}, x \in V_{n}$. Ясно, что $\pi^{\prime} \in Q_{n}, \eta^{\prime}(x) \in H_{n}$ и $\pi^{\prime} \cdot \eta^{\prime}=\sigma$. Поэтому $\left|Y_{n}\right| \leqslant 2^{2^{n-1}+n-1} \cdot n$ !

Докажем неравенство в другую сторону. Пусть $\sigma=\pi_{1} \cdot \eta_{1}=\pi_{2} \cdot \eta_{2}, \pi_{1}, \pi_{2} \in$ $Q_{n}, \eta_{1}, \eta_{2} \in H_{n}$. Тогда $\varepsilon=\sigma \cdot \sigma^{-1}=\pi_{1} \cdot \eta_{1} \cdot \eta_{2}^{-1} \cdot \pi_{2}^{-1}$, откуда $\pi_{1}^{-1} \cdot \pi_{2}=\eta_{1} \cdot \eta_{2}^{-1}$. Это означает, что $\pi_{1}^{-1} \cdot \pi_{2} \in Q_{n} \cap H_{n}=\left\{\varepsilon, 1_{n}\right\}$. Поэтому либо $\pi_{2}=\pi_{1}$, либо $\pi_{2}=\pi_{1} \widetilde{1}_{n}$. Аналогичным образом либо $\eta_{2}=\eta_{1}$, либо $\eta_{2}=\eta_{1} \widetilde{1}_{n}$. Поэтому $\left|Y_{n}\right| \geqslant 2^{2^{n-1}+n-1} \cdot n !$, откуда $\left|Y_{n}\right|=2^{2^{n-1}+n-1} \cdot n$ !

Теорема 10. При $n \geqslant 3$ для класса функиий $M_{7}(n)=\operatorname{Bi}(n) \cap C L(n)$ верно соотношение

$$
\mathfrak{G}_{\left\{M_{7}(n)\right\}}=Y_{n} .
$$

Доказательство. Включение $Q_{n}<\mathfrak{G}_{\left\{M_{7}(n)\right\}}$ очевидно. Кроме того, очевидно, что группа $\mathfrak{G}_{\left\{M_{7}(n)\right\}}$ транзитивна и инвариантна относительно разбиения пространства $V_{n}$ на блоки системы $\mathbb{B}$. Поэтому она импримитивна с этой системой блоков. Поскольку носитель каждой функции $f \in M_{7}(n)$ состоит из целого числа блоков, инверсия вектора внутри блока сохраняет эту функцию. Поэтому $H_{n}<\mathfrak{G}_{\left\{M_{7}(n)\right\}}$ и $Y_{n}<\mathfrak{G}_{\left\{M_{7}(n)\right\}}$. 
Докажем обратное включение. Для произвольной импримитивной группы $G<\mathfrak{G}$ с системой блоков $\mathbb{B}$ через $\widetilde{G}=\{\widetilde{g}, g \in G\}$ обозначим группу, задаваемую действием группы $G$ на $V_{n-1}$ по принципу: если $g \in G$ и $g\left(\Delta_{\alpha^{\prime}}\right)=\Delta_{\beta^{\prime}}$, то $\widetilde{g}\left(\alpha^{\prime}\right)=\beta^{\prime}$. Следуя [8], построим подстановочный гомоморфизм

$$
\xi:\left(\mathfrak{G}_{\left\{M_{7}(n)\right\}}, V_{n}\right) \rightarrow\left(\widetilde{\mathfrak{G}}_{\left\{M_{7}(n)\right\}}, V_{n-1}\right)
$$

по правилу $\xi(g)=\widetilde{g}$. Теперь заметим, что если $g \in \mathfrak{G}_{\left\{M_{7}(n)\right\}}$, то $\widetilde{g} \in$ $\mathfrak{G}_{\left\{M_{2}(n-1)\right\}}=A R_{n-1}^{(1)}$ по лемме 8. Таким образом, $\widetilde{\mathfrak{G}}_{\left\{M_{7}(n)\right\}}<A R_{n-1}^{(1)}$.

С другой стороны, для каждой подстановки $\widetilde{g} \in A R_{n-1}^{(1)}$ можно построить соответствующую подстановку $g \in \mathfrak{G}_{\left\{M_{7}(n)\right\}}$. Тогда по теореме 1.16 из [8] ядро $\operatorname{Ker} \xi$ состоит из всех подстановок из $\mathfrak{G}$, оставляющих на месте разбиение на блоки. Такие подстановки составляют группу $H_{n}$.

Следовательно, $\mathfrak{G}_{\left\{M_{7}(n)\right\}} / H_{n} \cong A R_{n-1}^{(1)}$. Осталось заметить, что $\left|T_{n}\right|=2^{2^{n-1}+n-1} \cdot n !=\left|A R_{n-1}^{(1)}\right| \cdot\left|H_{n}\right|$.

Теорема 11. При $n \geqslant 3$ для класса $M_{1}^{01}(n)=W P(n) \cap C L(n)$ выполняется равенство

$$
\mathfrak{G}_{\left\{M_{1}^{01}(n)\right\}}=\left\langle\widetilde{S}_{n}, H_{n}\right\rangle .
$$

Теорема 11 доказывается аналогично теореме 10 с учетом того факта, что $M_{1}^{01}(n) \subset M_{7}(n)$.

Перейдем к рассмотрению классов $M_{9}(n), M_{9}^{1}(n), \operatorname{Mon}^{\prime}(n), M_{10}(n), M_{10}^{0}(n)$ и $\overline{M_{\text {on }}^{\prime}}(n)$.

Tеорема 12. При $n \geqslant 3$ группы $\mathfrak{G}_{\left\{M_{9}(n)\right\}}, \mathfrak{G}_{\left\{M_{9}^{1}(n)\right\}}, \mathfrak{G}_{\left\{M o n^{\prime}(n)\right\}}, \mathfrak{G}_{\left\{M_{10}(n)\right\}}$, $\mathfrak{G}_{\left\{M_{10}^{0}(n)\right\}} u \mathfrak{G}_{\{\overline{M o n}(n)\}}$ coвnадают с группой $\widetilde{S}_{n}$.

Доказательство. Включение группы $\widetilde{S}_{n}$ в группы $\mathfrak{G}_{\left\{M_{9}(n)\right\}}, \mathfrak{G}_{\left\{M_{9}^{1}(n)\right\}}$, $\mathfrak{G}_{\left\{M o n^{\prime}(n)\right\}}, \mathfrak{G}_{\left\{M_{10}(n)\right\}}, \quad \mathfrak{G}_{\left\{M_{10}^{0}(n)\right\}}$ и $\mathfrak{G}_{\left\{\overline{M o n^{\prime}}(n)\right\}}$ очевидно. Докажем обратное включение. Заметим, что функциями веса $2^{n}-2$ в классах $M_{9}(n), M_{9}^{1}(n)$, $\operatorname{Mon}^{\prime}(n)$ являются только функции вида $h_{i}=x_{1} \vee \cdots \vee x_{i-1} \vee x_{i+1} \vee \cdots \vee x_{n}$, $i=\overline{1, n}$. Поэтому если $\sigma \in \mathfrak{G}_{\left\{M_{9}(n)\right\}}$ (либо $\sigma \in \mathfrak{G}_{\left\{M_{9}^{1}(n)\right\}}$, либо $\sigma \in \mathfrak{G}_{\left\{\operatorname{Mon}^{\prime}(n)\right\}}$ ), то $\sigma\left(E_{h_{i}}\right) \in\left\{E_{h_{1}}, \ldots, E_{h_{n}}\right\}$.

Соответственно, функциями веса $2^{n}-2$ в классах $M_{10}(n), \quad M_{10}^{0}(n)$, $\overline{M o n^{\prime}}(n)$ являются только функции вида $q_{i}=\bar{x}_{1} \vee \cdots \vee \bar{x}_{i-1} \vee \bar{x}_{i+1} \vee \cdots \vee \bar{x}_{n}$, $i=\overline{1, n}$. Поэтому если $\sigma \in \mathfrak{G}_{\left\{M_{10}(n)\right\}}$ (либо $\sigma \in \mathfrak{G}_{\left\{M_{10}^{0}(n)\right\}}$, либо $\sigma \in \mathfrak{G}_{\left\{\overline{M o n^{\prime}}(n)\right\}}$ ), то $\sigma\left(E_{q_{i}}\right) \in\left\{E_{q_{1}}, \ldots, E_{q_{n}}\right\}$. 
Проведем рассуждения для класса $M_{9}(n)$. Для остальных классов рассуждения аналогичны.

Пусть $\sigma=\left(s_{1}, \ldots, s_{n}\right) \in \mathfrak{G}_{\left\{M_{9}(n)\right\}}$. Положим $e_{1}=(1,0, \ldots, 0)$, $e_{2}=(0,1, \ldots, 0), \ldots, e_{n}=(0,0, \ldots, 1) \in V_{n}$. Единственной функцией веса $2^{n}-1$ в классе $M_{9}(n)$ является функция $g=x_{1} \vee \cdots \vee x_{n}$. Поэтому $\sigma\left(E_{g}\right)=E_{g}$, откуда следует, что $\sigma\left(0_{n}\right)=0_{n}$.

Покажем, что если $\alpha \in V_{n}$ и $\|\alpha\|=1$, то $\|\sigma(\alpha)\|=1$. В самом деле, функции $h_{i}, i=\overline{1, n}$, образуют совокупность орбит действия группы $\mathfrak{G}_{\left\{M_{9}(n)\right\}}$ на $P_{2}(n)$ как единственные функции веса $2^{n}-2$ в классе $M_{9}(n)$. Если $\alpha=e_{i}$, то $\alpha \notin E_{h_{i}}$ и $\alpha \in E_{h_{j}}, j \neq i$. Однако если $\|\sigma(\alpha)\|>1$, то вектор $\sigma(\alpha)$ входит во все носители $E_{h_{j}}, j=\overline{1, n}$, что невозможно из мощностных соображений, так как множества $E_{h_{j}}, j=\overline{1, n}$, равномощны и переходят одно в другое под действием подстановки $\sigma$.

Если же $\|\sigma(\alpha)\|=0$, то $0_{n}=\sigma(\alpha) \notin E_{h_{j}}, j=\overline{1, n}$, и опять получается противоречие. Таким образом, векторы $e_{1}, \ldots, e_{n}$ образуют орбиту группы $\mathfrak{G}_{\left\{M_{9}(n)\right\}}$ (то, что это именно орбита, а не объединение орбит, следует из включения $\left.\widetilde{S}_{n}<\mathfrak{G}_{\left\{M_{9}(n)\right\}}\right)$. Без ограничения общности будем считать, что $\sigma\left(e_{i}\right)=e_{i}, i=\overline{1, n}$.

Поскольку $x_{1}, \ldots, x_{n}, \bar{x}_{1}, \ldots, \bar{x}_{n} \in M_{9}(n)$, по лемме 1.6 из [1] (см. факт 1 из первого раздела) функции $s_{1}, \ldots, s_{n}, \bar{s}_{1}, \ldots, \bar{s}_{n}$ являются равновероятными функциями из класса $M_{9}(n)$. Рассмотрим функцию $s_{1}$. Возможны следующие варианты:

a) $s_{1} \in\left\{x_{1}, \ldots, x_{n}\right\}$

б) $s_{1} \in\left\{\bar{x}_{1}, \ldots, \bar{x}_{n}\right\}$,

в) $s_{1} \in\left\{x_{i} \oplus x_{j} \oplus 1,1 \leqslant i<j \leqslant n\right\}$,

г) $s_{1}-$ равновероятная монотонная функция, существенно зависящая не менее чем от трех переменных.

В силу задания класса $M_{9}(n)$ (см. табл. 1) других случаев быть не может. Случаи б)-в) невозможны, так как $\sigma\left(0_{n}\right)=0_{n}$. Рассмотрим случай г). Функция $s_{1}$ существенно зависит от $x_{1}$, так как $s_{1}\left(0_{n}\right)=0$ и $s_{1}\left(e_{1}\right)=1$. В этом случае, поскольку функция $s_{1}$ монотонна, она равна 1 на всех векторах вида $(1, *, \ldots, *)$. Но таких векторов ровно $2^{n-1}$, а $s_{1}$ - равновероятная монотонная функция. Следовательно, $s_{1}=x_{1}$. Остается только случай а). Проведя аналогичные рассуждения для функций $s_{2}, \ldots, s_{n}$, получим, что $\sigma \in \widetilde{S}_{n}$.

Следующая теорема завершает описание стабилизаторов классов функций из Галуа-замкнутых подалгебр алгебры Шефера от $n$ переменных. 
Теорема 13. При $n \geqslant 3, m \geqslant 2$ группы $\mathfrak{G}_{\left\{\operatorname{Mon}_{m}^{\prime}(n)\right\}}, \mathfrak{G}_{\left\{M_{9, m}(n)\right\}}, \mathfrak{G}_{\left\{W P_{m, 2}^{1}(n)\right\}}$, $\left.\mathfrak{G}_{\left\{\overline{M o n}{ }_{m}^{\prime}\right.}(n)\right\}, \mathfrak{G}_{\left\{M_{10, m}(n)\right\}}, \mathfrak{G}_{\left\{W N_{m, 2}^{0}(n)\right\}}$ coвnадают с группой $\widetilde{S}_{n}$.

Доказательство. Для групп $\mathfrak{G}_{\left\{M o n_{m}^{\prime}(n)\right\}}, \mathfrak{G}_{\left\{M_{9, m}(n)\right\}}, \mathfrak{G}_{\left\{\overline{M o n_{m}^{\prime}}(n)\right\}}$ и $\mathfrak{G}_{\left\{M_{10, m}(n)\right\}}$, а также $\mathfrak{G}_{\left\{W P_{m, 2}^{1}(n)\right\}}$ и $\mathfrak{G}_{\left\{W N_{m, 2}^{0}(n)\right\}}$ при $m \geqslant 3$ доказательство теоремы строится аналогично доказательству теоремы 12 путем рассмотрения функций максимального веса, отличных от константы. Такими функциями будут либо функции вида $x_{i_{1}} \vee \cdots \vee x_{i_{m}}$, либо функции вида $\bar{x}_{i_{1}} \vee \cdots \vee \bar{x}_{i_{m}}$, в зависимости от рассматриваемого класса.

Для групп $\mathfrak{G}_{\left\{W P_{2,2}^{1}(n)\right\}}$ и $\mathfrak{G}_{\left\{W N_{2,2}^{0}(n)\right\}}$ результат вытекает из теоремы А в [2], поскольку $W P_{2,2}^{1}(n)=B i^{1}(n)$ и $W N_{2,2}^{0}(n)=B i^{0}(n)$.

Объединяя результаты настоящей статьи с результатами из [1] и [2], получаем перечень стабилизаторов семейств функций, зависящих от $n$ переменных, из Галуа-замкнутых подалгебр алгебры Шефера.

Таблица 2

\begin{tabular}{|l|l|l|l|l|l|}
\hline № & Класс & Группа & № & Класс & Группа \\
\hline 1 & $P_{2}$ & $S_{2^{n}}$ & 24 & $W N$ & $\widetilde{S}_{n}$ \\
\hline 2 & $0-S$ & $S_{2^{n}-1}$ & 25 & $W N^{0}$ & $\widetilde{S}_{n}$ \\
\hline 3 & $1-S$ & $S_{2^{n}-1}$ & 26 & $W N^{1}$ & $\widetilde{S}_{n}$ \\
\hline 4 & $C L$ & $S_{2^{n-1}}$ & 27 & $W N^{01}$ & $\widetilde{S}_{n}$ \\
\hline 5 & $0-S \cap 1-S$ & $\bar{S}_{2^{n}-2}$ & 28 & $W N_{\infty, 2}$ & $\widetilde{S}_{n}$ \\
\hline 6 & $C L^{1}$ & $S_{2^{n-1}-1}$ & 29 & $W N_{m, 2}, m \geqslant 2$ & $\widetilde{S}_{n}$ \\
\hline 7 & $A$ & $A G L_{n}$ & 30 & $A^{01}$ & $L_{n-1} 2 S_{2}$ \\
\hline 8 & $A^{0}$ & $G L_{n}$ & 31 & $M_{8}$ & $A G L_{n-1} 2 S_{2}$ \\
\hline 9 & $A^{1}$ & $\left(A G L_{n}\right)_{n}$ & 32 & $M_{2}$ & $A R_{n}^{(1)}$ \\
\hline 10 & $B i$ & $Q_{n}$ & 33 & $M_{7}$ & $\left\langle Q_{n}, H_{n}\right\rangle$ \\
\hline 11 & $M_{3}$ & $C S_{n}$ & 34 & $M_{1}^{01}$ & $\left\langle\widetilde{S}_{n}, H_{n}\right\rangle$ \\
\hline 12 & $M_{3}^{0}$ & $\widetilde{S}_{n}$ & 35 & $M_{9}$ & $\widetilde{S}_{n}$ \\
\hline 13 & $M_{3}^{1}$ & $\widetilde{S}_{n}$ & 37 & $M_{9}^{1}$ & $\widetilde{S}_{n}$ \\
\hline 14 & $M_{3}^{01}$ & $\widetilde{C S}_{n}$ & $\widetilde{S}_{n}$ \\
\hline 15 & $M_{1}$ & $C S_{n}$ & 38 & $M o n_{m}^{\prime}, m \geqslant 2$ & $\widetilde{S}_{n}$ \\
\hline 16 & $M_{1}^{0}$ & $\widetilde{S}_{n}$ & 39 & $M_{9, m}, m \geqslant 2$ & $\widetilde{S}_{n}$ \\
\hline 17 & $M_{1}^{1}$ & $\widetilde{S}_{n}$ & 40 & $W P_{m, 2}^{1}, m \geqslant 2$ & $\widetilde{S}_{n}$ \\
\hline 18 & $W P$ & $\widetilde{S}_{n}$ & 41 & $M_{10}$ & $\widetilde{S}_{n}$ \\
\hline 19 & $W P^{0}$ & $\widetilde{S}_{n}$ & 42 & $M_{10}^{0}$ & $\widetilde{S}_{n}$ \\
\hline 20 & $W P^{1}$ & $\widetilde{S}_{n}$ & 43 & $\widetilde{M o n}^{\prime}$ & $\widetilde{S}_{n}$ \\
\hline 21 & $W P^{01}$ & $\widetilde{S}_{n}$ & $\widetilde{S o n}_{n}, m \geqslant 2$ & $\widetilde{S}_{n}$ \\
\hline 22 & $W P_{\infty, 2}$ & $\widetilde{S}_{n}$ & $M_{10, m}, m \geqslant 2$ & $\widetilde{S}_{n}$ \\
\hline 23 & $W P_{m, 2}, m \geqslant 2$ & & & $\widetilde{S}_{n}$ \\
\hline
\end{tabular}




\section{Список литературы}

[1] Горшков С.П., Тарасов А.В., “О максимальных группах инвариантных преобразований мультиаффинных, биюнктивных, слабо положительных и слабо отрицательных булевых функций”, Дискретная математика, 21 :2 (2009), 94-101.

[2] Тарасов А.В., “Стабилизаторы некоторых семейств булевых функций от $n$ переменных, образующих Галуа-замкнутые подалгебры алгебры Шефера”, Математические вопросы криптографии, 6:4 (2015), 79-105.

[3] Литвиненко В.С., Тарасов А. В., “Классы Шефера, классы Поста и соответствия Галуа”, Математические вопросы криптографии, 6:1 (2015), 81-108.

[4] Тарасов А. В., “Обобщение критерия биюнктивности Шефера”, Дискретная математика, $24: 2$ (2012), 91-99.

[5] Боднарчук В.Г., Калужнин Л.А., Котов В.Н., Ромов Б.А., “Теория Галуа для классов Поста”, Кибернетика, № 3 (1969), 1-10; № 5, 1-9.

[6] Марченков С.С., “Инварианты классов Поста", Фундам. и прикл. матем., 4:4 (1998), $1385-1404$

[7] Марченков С. С., Замкнутые классы булевых функиий, М. : Физматлит, 2001, 126 с.

[8] Погорелов Б. А., Основы теории групп подстановок. Ч.1. Общие вопросы, М., 1986.

[9] Калужнин Л.А., Клин М.Х., Сущанский В.И., “Экспоненцирование групп подстановок, I", Известия ВУЗов. Математика, № 8(207) (1979), 26-33.

[10] Погорелов Б.А., "Подметрики метрики Хемминга и теорема А.А. Маркова", Tpyды по дискретной математике, 9 (2006), 190-219.

[11] Горшков С.П., Тарасов А.В., Сложность решения систем булевых уравнений, М. : Курс, 2017, $192 \mathrm{c}$. 